Pacific Journal of Mathematics

THE SPACE OF ANR'S OF A CLOSED SURFACE 


\section{THE SPACE OF ANR's OF A CLOSED SURFACE}

\section{LAURENCE BOXER}

We study the hyperspace (denoted $2_{h}^{M}$ ) of ANR's of a (polyhedral) closed surface $M$. The topology of $2_{h}^{M}$ is induced by Borsuk's homotopy metric. We show the subpolyhedra of $M$ are dense in $2_{h}^{M}$. We obtain a necessary and sufficient condition for an arc in $2_{h}^{M}$ joining two points. We show that $2_{h}^{M}$ is an $\operatorname{ANR}(\mathscr{C})$. We prove that the subspace of $2_{h}^{M}$ whose members are AR's has the homotopy type of $M$.

o. Introduction. For a finite-dimensional compactum $X$ with metric $\rho$, let $2_{h}^{X}$ denote the space of nonempty compact ANR subsets of $X$. The topology of $2_{h}^{X}$ is induced by the metric $\rho_{h}$ defined by Borsuk [3]. In [1] and [2], Ball and Ford studied several properties of $2_{h}^{X}$, particularly for the case $X=S^{2}$. In this paper we generalize several of their results.

Throughout this paper, $M$ will denote a (polyhedral) closed surface. We show the nonempty polyhedral subcompacta of $M$ are dense in $2 M$. We give a necessary and sufficient condition for the existence of an arc in $2_{h}^{M}$ joining two given members of $2_{h}^{M}$. We show $2_{h}^{M}$ is an absolute neighborhood retract for metrizable spaces (ANR $(\mathscr{C})$ ) and that the subspace of $2_{h}^{M}$ whose members are the compact AR subsets of $M$ has the homotopy type of $M$.

Most of the results of this paper appeared in the author's doctoral thesis at the University of Illinois, Urbana-Champaign. The author wishes to thank his advisor, Mary-Elizabeth Hamstrom, for her guidance and encouragement. The author also wishes to thank B. J. Ball and the referee for several useful suggestions.

1. Preliminaries. Let $\rho$ be a metric for $M$. We use the following notation: If $x \in M$ and $A \subset M$, then

$$
B(x, r)=\{y \in M \mid \rho(x, y)<r\} ;
$$

$\bar{A}$, Int $A$, and $\operatorname{Bd} A$ are the closure, interior, and boundary of $A$ (in $M$ ) respectively.

Euclidean $n$-space is denoted $R^{n}$. The interval $[0,1]$ is denoted I. If $x, y \in R^{n}$ and $t \in R^{1}$, then $x+y$ will indicate the vector sum, and $t \cdot x$ will indicate scalar multiplication of $x$ by $t$.

If $A$ is a polyhedron, we will assume $A$ is compact unless otherwise stated.

A $\operatorname{map}$ is a continuous function.

We use the following notation and terminology of [1] and [2]: 
A $\delta$-set or a $\delta$-arc is a set or arc of diameter less than $\delta$. A $\delta$-map or a $\delta$-embedding is a map or embedding that moves no point by as much as $\delta$. The words "every $\delta$-subset of $A$ contracts to a point in an $\varepsilon$-subset of $A^{\prime \prime}$ are denoted $s(A, \delta, \varepsilon)$.

Where more than one topology is considered on a set, the topology in which a sequence converges will be indicated by an obvious notation. For example, $a_{n} \rightarrow a_{0}$ indicates that the sequence $\left\{a_{n}\right\}_{n=1}^{\infty}$ converges to $a_{0}$ in the topology of the metric $\rho$.

Let $X$ be a finite-dimensional compactum. Let $\rho$ be a metric for $X$. Let $A$ and $B$ be nonempty compact ANR subsets of $X$. The Hausdorff metric $\rho_{s}$ is given by

$$
\rho_{s}(A, B)=\max \{\sup \{\rho(a, B) \mid a \in A\}, \sup \{\rho(b, A) \mid b \in B\}\} \text {. }
$$

The homotopy metric $\rho_{h}$ is characterized in [3] by the following: Let $A$ and $\left\{A_{n}\right\}_{n=1}^{\infty}$ be nonempty compact ANR subsets of a finitedimensional compactum $X$. Then $A_{n} \underset{\rho_{h}}{\rightarrow} A$ if and only if

(a) $A_{n} \underset{\rho_{s}}{\rightarrow} A$, and

(b) given $\varepsilon>0$, there is a $\delta>0$ such that for all $n, s\left(A_{n}, \delta, \varepsilon\right)$.

We denote by $2_{h}^{X}$ the topological space whose members are the nonempty compact ANR subsets of $X$ and whose topology is induced by the metric $\rho_{h}$. It is shown in [3] that $2_{h}^{X}$ is complete and separable, and that $2_{h}^{X}$ is a topological invariant of $X$. We mention here other useful results of Borsuk: If $\rho_{h}(A, B)<\varepsilon$, then there are $\varepsilon$-maps $f: A \rightarrow B$ and $g: B \rightarrow A$. For $C \in 2_{h}^{X}$, let $[C]_{X}$ denote the collection of all members of $2_{h}^{X}$ that have the same homotopy type as $C$. Then $[C]_{X}$ is open in $2_{h}^{X}$. Since these sets partition $2_{h}^{X},[C]_{X}$ is also closed.

The terms homotopy, deformation retraction, isotopy, etc. will be used in standard fashion, except that it will be convenient not to insist that the interval be $I$. For example, if $c<d$, a deformation retraction of $A$ onto $B$ is a map $H: A \times[c, d] \rightarrow A$ such that $H_{c}=\operatorname{Id}_{A}$ and $H_{d}$ is a retraction of $A$ onto $B$. (We use the notation $H_{t}(a)=$ $H(a, t)$ for all $(a, t) \in A \times[c, d]$.) It will occasionally be convenient to refer to the map $H_{d}$ as a deformation retraction. A map $H: A \times$ $[c, d] \rightarrow A$ is strongly contracting if $c \leqq u \leqq v \leqq d$ implies $H_{u} \circ H_{v}(A) \subset$ $H_{v}(A) \subset H_{u}(A)([1]$, p. 37).

The term surface will be used to refer to a (second countable) connected 2-manifold, with or without boundary. A closed surface is a compact surface without boundary. A bounded surface is a compact surface with boundary. We differ from [1] and [2] in that we will call an annulus any space homeomorphic to $\left\{(x, y) \in R^{2} \mid 1 \leqq\right.$ $\left.x^{2}+y^{2} \leqq 2\right\}$.

The following gives a useful criterion for convergence in $2_{h}^{X}$ : 
Lemma 1.1 ([1], 3.4, p. 38). Let $A$ and $B$ be members of $2_{h}^{X}(X$ an arbitrary finite-dimensional compactum). Let $h: A \times I \rightarrow A$ be a strong deformation retraction of $A$ onto $B$. Let $\left\{t_{n}\right\}_{n=1}^{\infty}$ be an increasing sequence in $I$ converging to 1 . Suppose that for each $n, A_{n}=h_{t_{n}}(A)$ is an ANR. If

(a) $h$ is strongly contracting, or

(b) for all $n, h \mid A_{n} \times\left[t_{n}, t_{n+1}\right]$ is a strong deformation retraction of $A_{n}$ onto $A_{n+1}$, then $A_{n} \underset{\rho_{h}}{\longrightarrow} B$.

REMARKS. Case (b) above is not proved in [1], but the proof is identical to that of (a). We will use both cases.

The next two lemmas will be used in questions of arcs.

Lemma 1.2 ([1], 4.1, p. 43). If $A_{n} \overrightarrow{\rho_{h}} A$ in $2_{h}^{X}$ and if for each $n$ there is an $\varepsilon_{n}$-embedding $g_{n}: A_{n} \rightarrow X$ of $A_{n}$ into $X$, where $\varepsilon_{n} \rightarrow 0$, then $g_{n}\left(A_{n}\right) \underset{\rho_{h}}{\longrightarrow} A$.

Lemma 1.3 ([1], 4.2 and 4.3, p. 43). If $A \in 2_{h}^{X}$ and $f: A \times I \rightarrow X$ is an isotopy, then $\left\{f_{t}(A) \mid t \in I\right\}$ contains an arc in $2_{h}^{X}$ from $A$ to $f_{1}(A)$.

The next two results will be used several times:

THEOREM 1.4 ([11], 3.4, pp. 382-383). Let $N$ be a compact surface with $m$ boundary curves. Let $L$ be a closed surface containing disjoint open disks $D_{1}, \cdots, D_{m}$ such that $N=L \backslash \bigcup_{j=1}^{m} D_{j}$. Let $r: N \rightarrow N$ be a deformation retraction of $N$, and let $R=r(N)$. Then $L \backslash R$ is a union of $m$ simply-connected components $G_{1}, \cdots, G_{m}$, with $D_{j} \subset G_{j}$ for $j=1, \cdots, m$.

An immediate consequence of the above is:

CoROllary 1.5. Let $N$ be a bounded surface. Let $R \subset \operatorname{Int} N$ be a bounded surface that is a deformation retract of $N$. Then each component of $\overline{N \backslash R}$ is an annulus.

In the following theorems of Epstein, $N$ will denote a surface, with or without boundary, compact or not.

THEOREM 1.6 ([8], 1.7, p. 85). If a simple closed curve $S \subset N$ contracts to a point in $N$ then $S$ bounds a disk in $N$.

THEOREM 1.7 ([8], A2, p. 106) (stated in a different form). Sup- 
pose $N$ is a polyhedral surface and $f: I \rightarrow N$ is an embedding with $f^{-1}(\operatorname{Bd} N)=\{0,1\}$. Let $U$ be a neighborhood of $f(I)$ in $N$. Then there is an ambient isotopy of $N$ that is fixed on $\mathrm{Bd} N$ and outside $U$ and that changes $f$ to a piecewise linear embedding.

The following lemmas will be used in the next section.

Lemma 1.8. Let $Y$ be a topological space, $L \subset Y$, and let $\beta$ be an arc with endpoints $u$ and $v$ such that $\beta \subset L$. Suppose there is an open set $D$ in $Y \backslash\{u, v\}$ and an arc $\bar{\gamma} \subset L$ with endpoints $a$ and $b$ such that $\{a, b\} \subset \operatorname{Bd} D$ and $\gamma=\bar{\gamma} \backslash\{a, b\}$ is a component of $L \cap D$. Then either $\gamma \cap \beta=\phi$ or $\bar{\gamma} \subset \beta$.

Proof. Let $p:(I, 0,1) \rightarrow(\beta, u, v)$ be a homeomorphism. (The notation means that $p$ is a map from $I$ to $\beta$ such that $p(0)=u$ and $p(1)=v$.) Suppose $\gamma \cap \beta \neq \phi$. There is an $x \in \gamma$ and a $t_{0} \in(0,1)$ such that $p\left(t_{0}\right)=x$. Then $A=p^{-1}(\beta \cap D)$ is a nonempty open set in $I$ contained in $(0,1)$. Thus $t_{0}$ lies in a component $\left(a_{0}, b_{0}\right)$ of $A$. We have $x \in p\left(\left(a_{0}, b_{0}\right)\right) \subset \beta \cap D \subset L \cap D$, so $p\left(\left(a_{0}, b_{0}\right)\right)$ is a connected subset of $L \cap D$ containing $x$. Thus $p\left(\left(a_{0}, b_{0}\right)\right) \subset \gamma$ and $\left\{p\left(a_{0}\right), p\left(b_{0}\right)\right\} \cap D=\phi$, so $\left\{p\left(a_{0}\right), p\left(b_{0}\right)\right\} \subset \mathrm{Bd} D$. The arc $B=p\left(\left[a_{0}, b_{0}\right]\right)$ has its interior in $\gamma$, but the endpoints of $B$ are not in $\gamma$. Therefore $\bar{\gamma}=B \subset p(I)=\beta$.

The following is an immediate consequence of ([7], 4.2, p. 360):

LEMmA 1.9. If $A$ is an annulus with boundary curves $T_{1}$ and $T_{2}$, let $H: T_{2} \times I \rightarrow A$ be a map such that $H_{0}=\operatorname{Id}_{T_{2}}$ and $H_{1}\left(T_{2}\right)=T_{1}$. Then $H\left(T_{2} \times I\right)=A$.

We say $Y$ dominates $X$ if there are maps $f: X \rightarrow Y$ and $g: Y \rightarrow X$ such that $g \circ f$ is homotopic to $\operatorname{Id}_{X}$. We write $\Delta X=\min \{\operatorname{dim} Y / Y$ is a finite simplicial complex that dominates $X$ \}.

2. The role of the polyhedra. In [3], Borsuk asked the following questions: If $X$ is a polyhedron, is the collection of all nonempty subpolyhedra of $X$ dense in $2_{h}^{X}$ ? What is the category (in the sense of Baire) of the collection of all nonempty subpolyhedra of $X$ in $2_{h}^{X}$ ? In [1], the first question was answered affirmatively for the case $X=S^{2}$, and the second question was given the following answer: If $X$ is a connected polyhedron with no 1-dimensional open subset, the collection of all nonempty polyhedra properly contained in $X$ is a first category subset of $2_{h}^{X}$. It was also shown in [1] that the collection of nonempty topological polyhedra (i.e., homeomorphic images of polyhedra) properly contained in $S^{2}$ is a dense $G_{\delta}$, hence 
second category, subset of $2_{h}^{S^{2}}$. We will extend the above to closed surfaces.

Lemma 2.1. If $X$ is a finite-dimensional compactum and $U$ is open in $X$, then $\mathscr{Q}_{b}=\left\{C \in 2_{h}^{X} \mid C \subset U\right\}$ is open in $2_{h}^{X}$.

Proof. Let $\left\{A_{n}\right\}_{n=1}^{\infty} \subset 2_{h}^{X} \backslash \mathscr{L}$. Assume $A_{n} \rightarrow A_{\rho_{h}}$. For each $n$ there exists $x_{n} \in A_{n} \backslash U$. Since $X$ is compact we may assume (by taking a subsequence if necessary) that $x_{n} \rightarrow x_{0} \in X \backslash U$. Since $A_{n} \underset{\rho_{\mathbf{g}}}{\rightarrow} A_{0}$, we have $x_{0} \in A_{0}$. Therefore $A_{0} \notin \mathscr{U}$, so $\mathscr{U}$ is open.

We prove a theorem about the Baire category of the collection of topological polyhedra in $M$ as a subset of $2_{h}^{M}$. (Recall $M$ is a (polyhedral) closed surface.)

THEOREM 2.2. Let $\mathscr{T}$ be the collection of nonempty topological polyhedra properly contained in $M$. Then $\mathscr{T}$ is a second category subset of $2_{h}^{M}$.

Proof. Let $D$ be a disk contained in $M$. By 2.1, $\mathscr{\mathscr { C }}=$ $\left\{Y \in 2_{h}^{M} \mid Y \subset \operatorname{Int} D\right\}$ is open in $2_{h}^{M}$, and thus is topologically complete. Let $f$ : Int $D \rightarrow S^{2}$ be an embedding. Then the map $f_{*}: \mathbb{Z} \rightarrow 2_{h}^{S^{2}}$ given by $f_{*}(Y)=f(Y)$ is an open embedding ([3], p. 198). Since the collection of nonempty topological polyhedra contained in $S^{2}$ is a dense $G_{o}$ subset of $2_{h}^{S^{2}}([1], 3.12$, p. 42), it follows that $\mathscr{Q} \backslash \mathscr{T}$ is a first category subset of $\mathscr{U}$. The classical Baire category theorem implies $\mathscr{Q} \cap \mathscr{T}$ is a second category subset of $\mathscr{U}$, and thus of $2_{h}^{M}$. Hence $\mathscr{T}$ is a second category subset of $2_{h}^{M}$.

The rest of this section is devoted to proving the following:

THEOREM 2.3. The collection of nonempty subpolyhedra of $M$ is dense in $2_{h}^{M}$.

To prove 2.3, we show in 2.4 that for a given $C \in 2_{h}^{M}$ we can split $M$ into two pieces that join along simple closed curves such that the intersection of $C$ with each piece is an ANR. Each of the pieces of $M$ embeds in $S^{2}$. In 2.5, we use the fact that the result is known for $S^{2}$ to construct a sequence of polyhedra whose intersection is $C$ satisfying the hypotheses of 1.1 .

LEMMA 2.4. Let $q$ be a positive integer. Assume $M$ is orientable with genus $q$ or nonorientable with genus $2 q$. Let $C \in 2_{h}^{M}$. Then there are compact subsurfaces $X_{1}$ and $X_{2}$ of $M$ and simple closed curves $\alpha_{1}, \cdots, \alpha_{q+1}$ in $M$ such that: 
(a) $M=X_{1} \cup X_{2}$.

(b) The $\alpha_{n}$ are pairwise disjoint.

(c) $\mathrm{Bd} X_{1}=\mathrm{Bd} X_{2}=X_{1} \cap X_{2}=\bigcup_{n=1}^{q+1} \alpha_{n}$.

(d) $X_{1}$ and $X_{2}$ both are homeomorphic to a sphere with $q+1$ disjoint open disks removed.

(e) $\bigcup_{n=1}^{q+1} \alpha_{n} \backslash C$ has finitely many components.

Proof. It is an easy consequence of the standard way to represent a surface that there are subsurfaces $X_{1}^{\prime}$ and $X_{2}^{\prime}$ of $M$ and simple closed curves $\alpha_{1}^{\prime}, \cdots, \alpha_{q+1}^{\prime}$ in $M$ satisfying (a) through (d). It follows that for each $n$ there is a two-sided collar $N_{n}$ of $\alpha_{n}^{\prime}$ in $M$ such that the $N_{n}$ are pairwise disjoint. For any $n$ such that $\alpha_{n}^{\prime} \backslash C$ has finitely many components, set $\alpha_{n}=\alpha_{n}^{\prime}$. Thus we suppose $\alpha^{\prime}$ is any of the $\alpha_{n}^{\prime}$ such that $\alpha_{n}^{\prime} \backslash C$ has infinitely many components. We write $N=N_{n}$. Clearly we may write $\alpha^{\prime} \backslash C=\bigcup_{m=1}^{\infty} \gamma_{m}$, where the $\gamma_{m}$ are distinct components of $\alpha^{\prime} \backslash C$ and each $\bar{\gamma}_{m}$ is an arc whose endpoints $a_{m}$ and $b_{m}$ lie in $C$.

Let $Z=\lim \sup \left\{\bar{\gamma}_{m}\right\}_{m=1}^{\infty}$, i.e., $Z$ is the set of all $x \in \alpha^{\prime}$ such that every neighborhood of $x$ meets infinitely many $\bar{\gamma}_{m}$. Then $Z$ is closed (see [13], p. 10). Thus $Z$ is a compact subset of $\alpha^{\prime}$. It is easily seen that $Z \subset C$.

Let $w_{0}, w_{1}$, and $w_{2}$ be distinct points of $\gamma_{1}$ such that $w_{0}$ lies in the arc $\overline{w_{1} w_{2}}$ of $\gamma_{1}$ from $w_{1}$ to $w_{2}$. Let $f_{0}:(I, 0,1) \rightarrow\left(\alpha^{\prime} \backslash\left(w_{1} w_{2} \mid\left\{w_{1}, w_{2}\right\}\right)\right.$, $w_{1}, w_{2}$ ) be a homeomorphism. Since $N$ is an annulus,

(1) there is a disk $B \subset N$ such that $N \backslash B$ is homeomorphic to $I \times(0,1), w_{0} \in(N \backslash B) \cap \alpha^{\prime} \subset \overline{N \backslash B} \cap \alpha^{\prime} \subset \gamma_{1}$, and $Z \cup f_{0}(I) \subset$ Int $B$. Since ANR's are locally arcwise connected, (1) implies that for each $z \in Z$ there is a neighborhood $U$ of $z$ contained in $\operatorname{Int} B$ such that $U \cap C$ is arcwise connected. Since $Z$ is compact,

(2) there are open sets $U_{1}, \cdots, U_{p}$ such that $Z \subset \bigcup_{k=1}^{p} U_{k} \subset \operatorname{Int} B$ and each $U_{k} \cap C$ is arcwise connected.

It is easily seen that for almost all $m$ there is a $k$ such that $\bar{\gamma}_{m} \subset U_{k}$. We assume $\bar{\gamma}_{1}, \cdots, \bar{\gamma}_{m_{0}}$ are those $\bar{\gamma}_{m}$ that fail to lie in any $U_{k}$. Define $\Gamma_{0}=\phi$, and for $k \in\{0,1, \cdots, p-1\}$ define

$$
\Gamma_{k+1}=\left\{\bar{\gamma}_{m} \subset U_{k+1} \mid \bar{\gamma}_{m} \notin \bigcup_{j=0}^{k} \Gamma_{j}\right\} .
$$

Define $\Gamma_{p+1}=\left\{\bar{\gamma}_{1}, \cdots, \bar{\gamma}_{m_{0}}\right\}$. For each $j$ let $\Gamma_{j}^{\prime}=\left\{\gamma_{m} \mid \bar{\gamma}_{m} \in \Gamma_{j}\right\}$. Clearly $\Gamma_{0}, \Gamma_{1}, \cdots, \Gamma_{p+1}$ partition $\left\{\bar{\gamma}_{m}\right\}_{m=1}^{\infty}$. Let the endpoints $a_{m}$ and $b_{m}$ of $\bar{\gamma}_{m}$ satisfy $f_{0}^{-1}\left(a_{m}\right)<f_{0}^{-1}\left(b_{m}\right)$. For $m>1, \bar{\gamma}_{m}=f_{0}\left(\left[f_{0}^{-1}\left(a_{m}\right), f_{0}^{-1}\left(b_{m}\right)\right]\right)$.

We begin an induction argument by observing that for $k=0$ we have a map $f_{k}:(I, 0,1) \rightarrow\left(\operatorname{Int} B, w_{1}, w_{2}\right)$ such that:

(3) If $t \in I$ and $f_{k}(t) \notin C$ then $f_{k}(t)=f_{0}(t)$. 


\section{(4) $f_{k}(I) \backslash C$ is a union of members of $\bigcup_{j=k+1}^{p+1} \Gamma_{j}^{\prime}$.}

Suppose for some $k<p, f_{k}:(I, 0,1) \rightarrow\left(\operatorname{Int} B, w_{1}, w_{2}\right)$ is a map satisfying (3) and (4). If $f_{k}(I) \backslash C$ meets no member of $\Gamma_{k+1}^{\prime}$ we define $f_{k+1}=f_{k}$; then (3) and (4) are satisfied when $k$ is replaced by $k+1$. Otherwise we define $c_{k}=\inf \left\{t \in I \mid f_{k}(t)\right.$ belongs to a member of $\left.\Gamma_{k+1}^{\prime}\right\}$, and $d_{k}=\sup \left\{t \in I \mid f_{k}(t)\right.$ belongs to a member of $\left.\Gamma_{k+1}^{\prime}\right\}$. By (4) and our choice of $\left\{w_{1}, w_{2}\right\}, 0<c_{k}<d_{k}<1$. By (3) and (4), each of $f_{k}\left(c_{k}\right)=$ $f_{0}\left(c_{k}\right)$ and $f_{k}\left(d_{k}\right)=f_{0}\left(d_{k}\right)$ must be an endpoint of some $\bar{\gamma}_{m} \in \Gamma_{k+1}$ or a member of $Z$. It follows that $\left\{f_{k}\left(c_{k}\right), f_{k}\left(d_{k}\right)\right\} \subset \overline{U_{k+1}} \cap C$.

If $\left\{f_{k}\left(c_{k}\right), f_{k}\left(d_{k}\right)\right\} \subset U_{k+1}$ then (2) implies there is an arc $\gamma_{k}^{\prime}$ in $U_{k+1} \cap C$ from $f_{k}\left(c_{k}\right)$ to $f_{k}\left(d_{k}\right)$.

If, say, $f_{k}\left(c_{k}\right) \notin U_{k+1}$ then there must be infinitely many members of $\Gamma_{k+1}^{\prime}$ that meet $f_{k}(I)$, for otherwise (4) implies $f_{k}\left(c_{k}\right)$ is an endpoint $a_{m}$ of some $\bar{\gamma}_{m} \in \Gamma_{k+1}$ and thus $f_{k}\left(c_{k}\right) \in U_{k+1}$, contrary to assumption. Thus $f_{k}\left(c_{k}\right) \in Z \cap U_{k_{1}}$ for some $k_{1}$. There is a sequence $\left\{a_{m_{r}}\right\}$ of endpoints of members $\overline{\gamma_{m_{r}}}$ of $\Gamma_{k+1}$ such that $f_{k_{k}} \circ f_{0}^{-1}\left(\overline{\gamma_{m_{r}}}\right) \not \subset C$ and $a_{m_{r}} \rightarrow f_{k_{k}}\left(c_{k}\right)$. Hence there is an $r$ such that $a_{m_{r}} \in U_{k_{1}}$. By (2) there are arcs $\gamma^{\prime}$ in $U_{k_{1}} \cap C$ from $f_{k}\left(c_{k}\right)$ to $a_{m_{r}}$ and $\gamma^{\prime \prime}$ in $U_{k+1} \cap C$ from $a_{m_{r}}$ to $f_{k}\left(d_{k_{k}}\right)$. There is an arc $\gamma_{k}^{\prime} \subset \gamma^{\prime} \cup \gamma^{\prime \prime} \subset C \cap \operatorname{Int} B$ from $f_{k}\left(c_{k}\right)$ to $f_{k}\left(d_{k}\right)$.

The other cases are treated as above. So in any case, $C \cap \operatorname{Int} B$ contains an arc $\gamma_{k}^{\prime}$ from $f_{k}\left(c_{k}\right)$ to $f_{k}\left(d_{k}\right)$. Let $f_{k+1}:(I, 0,1) \rightarrow\left(\operatorname{Int} B, w_{1}, w_{2}\right)$ be determined by: $f_{k+1} \mid\left[c_{k}, d_{k}\right]$ is a homeomorphism of $\left(\left[c_{k}, d_{k}\right], c_{k}, d_{k}\right)$ onto $\left(\gamma_{k}^{\prime}, f_{k}\left(c_{k}\right), f_{k}\left(d_{k}\right)\right)$; and $f_{k+1}(t)=f_{k}(t)$ for $t \in I \backslash\left[c_{k}, d_{k}\right]$. Clearly $f_{k+1}$ is continuous. The construction shows (3) and (4) are satisfied when $k$ is replaced by $k+1$.

With the induction completed, we have by (4) a map $f_{p}:(I, 0,1) \rightarrow$ (Int $B, w_{1}, w_{2}$ ) such that $f_{p}(I) \backslash C$ is a union of members of the finite set $\Gamma_{p+1}^{\prime}$. Now $f_{p}(I)$ contains an $\operatorname{arc} \beta$ from $w_{1}$ to $w_{2}$. Let $\gamma_{m}$ be a component of $f_{p}(I) \backslash C$. Apply 1.8, with $Y=M, L=f_{p}(I), D=$ $M \backslash\left(C \cup\left\{w_{1}, w_{2}\right\}\right), \bar{\gamma}=\bar{\gamma}_{m}$ : We have $\bar{\gamma}_{m} \subset \beta$ or $\gamma_{m} \cap \beta=\phi$. Therefore $\beta \backslash C$ has finitely many components, and $\alpha=\beta \cup \overline{w_{1} w_{2}}$ is a simple closed curve such that $\alpha \backslash C$ has finitely many components.

Let $h:$ Int $B \rightarrow R^{2}$ be a homeomorphism. Let $h^{\prime}:(I, 0,1) \rightarrow\left(\beta, w_{1}, w_{2}\right)$ be a homeomorphism. Let $g:([-1,1], 0,\{-1,1\}) \rightarrow\left(\alpha^{\prime}, w_{1},\left\{w_{2}\right\}\right)$ be a relative homeomorphism such that $g(I) \subset \operatorname{Int} B$. Define $H: \alpha^{\prime} \times I \rightarrow$ Int $N$ by

$$
H(g(s), t)=\left\{\begin{array}{l}
g(s) \quad \text { if } \quad-1 \leqq s \leqq 0 \\
h^{-1}\left[(1-t) \cdot h \circ g(s)+t \cdot h \circ h^{\prime}(s)\right] \text { if } \quad 0 \leqq s \leqq 1
\end{array}\right.
$$

Clearly $H$ is well-defined and continuous, $H_{0}=\mathrm{Id}_{\alpha^{\prime}}$, and $H_{1}$ is a homeomorphism of $\alpha^{\prime}$ onto $\alpha$. It follows from ([7], 2.1, p. 87) that there is a homeomorphism $T: N \rightarrow N$ such that $T\left(\alpha^{\prime}\right)=\alpha$ and $T(x)=x$ for all $x \in \operatorname{Bd} N$. 
By applying this construction to each of the curves $\alpha_{n}^{\prime}$, we easily obtain a homeomorphism $P: M \rightarrow M$ taking $X_{1}^{\prime}, X_{2}^{\prime}, \alpha_{1}^{\prime}, \cdots, \alpha_{q+1}^{\prime}$ onto sets satisfying (a) through (e).

Theorem 2.3 follows from 1.1 and the following:

THEOREM 2.5. Let $C \in 2_{h}^{M}$ be a proper subset of $M$. Then there is a sequence $\left\{A_{n}\right\}_{n=1}^{\infty}$ in $2_{h}^{M}$ such that for all $n$ :

(a) Each component of $A_{n}$ is a polyhedral bounded surface.

(b) $C \subset A_{n+1} \subset \operatorname{Int} A_{n}$.

Also there is a sequence $0=t_{1}<t_{2}<t_{3}<\cdots$ with $\lim t_{n}=1$ and a map $h: A_{1} \times I \rightarrow A_{1}$ such that:

(c) $h$ is a strong deformation retraction of $A_{1}$ onto $C$.

(d) For each $n, h \mid A_{n} \times\left[t_{n}, t_{n+1}\right]$ is a strong deformation retraction of $A_{n}$ onto $A_{n+1}$.

Proof. We remark that the proof is long, so some of the technical details have been omitted. A more complete proof is in [5].

It is easy to see that there is no loss of generality in assuming $C$ is connected. By sewing a Moebius band onto the boundary of a disk cut out of $M \backslash C$ if necessary, we can also assume that $M$ is nonorientable of even genus, or orientable. In view of $([1], 3.2,3.3$, and 3.5 , pp. 36-39) we assume $M \neq S^{2}$.

For a given connected $C \in 2_{h}^{M}$ with $C \neq M$, let $\alpha_{1}, \cdots, \alpha_{q+1}$, $N_{1}, \cdots, N_{q+1}, X_{1}, X_{2}$ be as in 2.4 and its proof. It follows from 2.4(e) and ([4], 2.12, p. 102) that $\hat{X}_{1}=X_{1} \cap C$ and $\hat{X}_{2}=X_{2} \cap C$ are ANR's. We may assume $\hat{X}_{1} \neq \phi$. For $k=1,2, X_{k} \cup \bigcup_{j=1}^{q+1} N_{j}$ is homeomorphic to $X_{k}$, which is embeddable in $S^{2}$. If $\hat{X}_{2} \subset \operatorname{Int}\left(\bigcup_{j=1}^{q+1} N_{j}\right)$ then $C \subset$ Int $\left(X_{1} \cup \bigcup_{j=1}^{q+1} N_{j}\right)$, in which case we are done, by [1]. Thus we assume

(1) $\hat{X}_{2} \not \subset \operatorname{Int}\left(\bigcup_{j=1}^{q+1} N_{j}\right)$.

Let $\Gamma$ be the set of components $\gamma$ of $\bigcup_{j=1}^{q+1} \alpha_{j} \backslash C$ such that $\gamma \subset \alpha_{j}$ implies $\gamma \neq \alpha_{j}$. From 2.4(e), $\Gamma$ is a finite set. We argue by induction on the number of members of $\Gamma$.

If $\Gamma=\phi$ then for each $j \in\{1,2, \cdots, q+1\}$ either $\alpha_{j} \subset C$ or $\alpha_{j} \subset$ $M \backslash C$. Since $C$ is connected and $\hat{X}_{1} \neq \phi$, if no $\alpha_{j}$ lies in $C$ we have $C=\hat{X}_{1}$, contrary to (1). We assume

(2) $\bigcup_{j=1}^{p} \alpha_{j} \subset C$ for some $p$ with $1 \leqq p \leqq q+1$, and if $p<q+1$ then $\bigcup_{j=p+1}^{q+1} \alpha_{j} \subset M \backslash C$.

Neither $\hat{X}_{1}$ nor $\hat{X}_{2}$ need be connected; nevertheless, the theorems of [1] cited above (and their proofs) imply there are sequences $\left\{B_{n}^{k}\right\}_{n=1}^{\infty}(k=1,2)$ such that for all $n$ :

(3) Each component of $B_{n}^{k}$ is a polyhedral surface.

(4) $\hat{X}_{k} \subset B_{n+1}^{k} \subset \operatorname{Int} B_{n}^{k} \subset B_{n}^{k} \subset \operatorname{Int}\left(X_{k} \cup \bigcup_{j=1}^{q+1} N_{j}\right)$. Also there are 
maps $h^{k}: B_{1}^{k} \times I \rightarrow B_{1}^{k}$ and a sequence $0=t_{1}<t_{2}<t_{3}<\cdots$ such that $\lim t_{n}=1$,

(5) $h^{k}$ is a strong deformation retraction of $B_{1}^{k}$ onto $\hat{X}_{k}$, and for each $n$ :

(6) $h^{k} \mid B_{n}^{k} \times\left[t_{n}, t_{n+1}\right]$ is a strong deformation retraction of $B_{n}^{k}$ onto $B_{n+1}^{k}$.

(7) $h^{k} \mid\left(\mathrm{Bd} B_{n}^{k}\right) \times\left[t_{n}, t_{n+1}\right]$ is an isotopy of $\mathrm{Bd} B_{n}^{k}$ onto $\mathrm{Bd} B_{n+1}^{k}$.

(8) If $y \in \mathrm{Bd} B_{n}^{k}$ and $x \in h^{k}\left(\{y\} \times\left[t_{n}, t_{n+1}\right]\right)$, then $h^{k}\left(\{x\} \times\left[t_{n}, t_{n+1}\right]\right) \subset$ $h^{k}\left(\{y\} \times\left[t_{n}, t_{n+1}\right]\right)$ and $h^{k}(x, t)=h^{k}(y, t)$ for $t \in\left[t_{n+1}, 1\right]$.

(9) For all $x \in \mathrm{Bd} B_{n}^{k}, h^{k}(\{x\} \times I)$ is an arc and $h^{k}(\{x\} \times[0,1))$ is a (noncompact) polyhedron.

(10) If $D$ is a component of $B_{n}^{k} \backslash \hat{X}_{k}$ and $E$ is a component of Bd $D$ such that $E \subset \hat{X}_{k}$, then there is a boundary curve $\beta$ of $B_{n}^{k}$ such that $\beta \subset D$ and $h_{1}^{k}(\beta)=E$.

From (2) and (4) we may assume for all $n$ and for $k=1,2$,

(11) $\bigcup_{j=1}^{p} \alpha_{j} \subset \operatorname{Int} B_{n}^{k}$ and $B_{n}^{k} \cap \bigcup_{j=p+1}^{q+1} \alpha_{j}=\phi$.

For all $n$, let $A_{n}=\left(B_{n}^{1} \cap X_{1}\right) \cup\left(B_{n}^{2} \cap X_{2}\right)$. We define a map $h$ on $A_{1} \times I$ by

$$
h(x, t)=\left\{\begin{array}{lll}
h^{1}(x, t) & \text { if } & x \in B_{1}^{1} \cap X_{1} \\
h^{2}(x, t) & \text { if } & x \in B_{1}^{2} \cap X_{2}
\end{array}\right.
$$

If $x \in\left(B_{1}^{1} \cap X_{1}\right) \cap\left(B_{1}^{2} \cap X_{2}\right)=\bigcup_{j=1}^{p} \alpha_{j}=\hat{X}_{1} \cap \hat{X}_{2}$, then (5) implies $h^{1}(x, t)=$ $x=h^{2}(x, t)$ for all $t \in I$. Therefore $h$ is well-defined and continuous. It is easily seen that

(12) if $x \in B_{1}^{k} \cap X_{k}$ then $h(x, t) \in B_{1}^{k} \cap X_{k}$. It follows that $h\left(A_{1} \times I\right)=A_{1}$.

By (11), if $\beta$ is a boundary curve of $B_{n}^{k}$ then $\beta \subset \operatorname{Int} X_{1}$ or $\beta \subset$ Int $X_{2}$. The union of those boundary curves of $B_{n}^{k}$ that lie in Int $X_{k}$ is $\left(\mathrm{Bd} A_{n}\right) \cap X_{k}$. It follows that $A_{n}$ is a polyhedral bounded surface.

For all $n, C \subset A_{n+1}=\left(B_{n+1}^{1} \cap X_{1}\right) \cup\left(B_{n+1}^{2} \cap X_{2}\right) \subset\left[\left(\operatorname{Int} B_{n}^{1}\right) \cap X_{1}\right] \cup$ $\left[\left(\operatorname{Int} B_{n}^{2}\right) \cap X_{2}\right]=\operatorname{Int}\left(B_{n}^{1} \cap X_{1}\right) \cup \bigcup_{j=1}^{p} \alpha_{j} \cup \operatorname{Int}\left(B_{n}^{2} \cap X_{2}\right)=\operatorname{Int} A_{n}$.

It is clear that $h_{0}=\operatorname{Id}_{A_{1}}$ and $h_{t} \mid C=\operatorname{Id}_{C}$ for all $t \in I$. Also $h_{1}\left(A_{1}\right)=$ $h_{1}^{1}\left(B_{1}^{1} \cap X_{1}\right) \cup h_{1}^{2}\left(B_{1}^{2} \cap X_{2}\right)=\left(\right.$ by (5) and (12)) $\hat{X}_{1} \cup \hat{X}_{2}=C$. Thus $h$ is a strong deformation retraction of $A_{1}$ onto $C$.

For all $n$, we see by (6) and (12) that $h \mid A_{n} \times\left[t_{n}, t_{n+1}\right]$ is a strong deformation retraction of $A_{n}$ onto $A_{n+1}$.

By (12), analogues of (7) through (9) hold when we replace $\left(\hat{X}_{k},\left\{B_{n}^{k}\right\}_{n=1}^{\infty}, h^{k}\right)$ with $\left(C,\left\{A_{n}\right\}_{n=1}^{\infty}, h\right)$.

If $D$ is a component of $A_{n} \mid C$ then by (11) $D$ is a component of $B_{n}^{k} \backslash \hat{X}_{k}$ for some $k$. Then (10) and the construction imply $\left(C,\left\{A_{n}\right\}_{n=1}^{\infty}, h\right)$ satisfies the analogue of (10). This concludes our discussion of the case $\Gamma=\phi$.

Suppose the theorem is true whenever $\Gamma$ has less than $r$ members 
$(r>0)$. Now let $\Gamma$ have $r$ distinct members, $\gamma_{1}, \cdots, \gamma_{r}$. Topologically $\gamma_{r}$ is an open interval in some $\alpha_{j}$, say $\gamma_{r} \subset \alpha_{1}$. Let $\left\{z_{1}, z_{2}\right\}$ be the endpoints of $\gamma_{r}\left(z_{1}=z_{2}\right.$ if $\left.\bar{\gamma}_{r}=\alpha_{1}\right)$. Let $C^{\prime}=C \cup \bar{\gamma}_{r}$. Clearly $C^{\prime}$ is a connected ANR, and $\Gamma^{\prime}=\left\{\gamma_{1}, \cdots, \gamma_{r-1}\right\}$ is the set of all components $\gamma$ of $\bigcup_{j=1}^{q+1} \alpha_{j} \backslash C^{\prime}$ such that $\gamma \subset \alpha_{j}$ implies $\gamma \neq \alpha_{j}$. The inductive hypothesis gives a sequence $\left\{B_{n}\right\}_{n=1}^{\infty} \subset 2_{h}^{M}$ such that for all $n$ :

(13) $B_{n}$ is a polyhedral bounded surface.

(14) $C^{\prime} \subset B_{n+1} \subset \operatorname{Int} B_{n}$.

Also there is a map $\psi: B_{1} \times I \rightarrow B_{1}$ and a sequence $0=t_{1}<t_{2}<t_{3}<\cdots$ such that $\lim t_{n}=1$,

(15) $\psi$ is a strong deformation retraction of $B_{1}$ onto $C^{\prime}$, and for all $n$ :

(16) $\psi / B_{n} \times\left[t_{n}, t_{n+1}\right]$ is a strong deformation retraction of $B_{n}$ onto $B_{n+1}$.

(17) $\psi /\left(\mathrm{Bd} B_{n}\right) \times\left[t_{n}, t_{n+1}\right]$ is an isotopy of $\mathrm{Bd} B_{n}$ onto $\mathrm{Bd} B_{n+1}$.

(18) If $y \in \mathrm{Bd} B_{n}$ and $x \in \psi\left(\{y\} \times\left[t_{n}, t_{n+1}\right]\right)$ then $\psi\left(\{x\} \times\left[t_{n}, t_{n+1}\right]\right) \subset$ $\psi\left(\{y\} \times\left[t_{n}, t_{n+1}\right]\right)$ and $\psi(x, t)=\psi(y, t)$ for $t \in\left[t_{n+1}, 1\right]$.

(19) For all $x \in \mathrm{Bd} B_{n}, \psi(\{x\} \times I)$ is an arc and $\psi(\{x\} \times[0,1))$ is a (noncompact) polyhedron.

(20) If $D$ is a component of $B_{n} \backslash C^{\prime}$ and $E$ is a component of Bd $D$ such that $E \subset C^{\prime}$, then there is a boundary curve $\beta$ of $B_{n}$ such that $\beta \subset D$ and $\psi_{1}(\beta)=E$.

For all $n$ we define $\varepsilon_{n}=\sup \left\{\operatorname{diam} \psi(\{x\} \times I) / x \in B_{n}\right\}$. By compactness, $\varepsilon_{n}$ is finite, and we easily see

(21) $\lim \varepsilon_{n}=0$.

Let $D$ be a component of $B_{1} \backslash C^{\prime}$ such that $\bar{\gamma}_{r}$ lies in a boundary component $E$ of $D$. From (20) there is a boundary curve $\beta$ of $B_{1}$ such that $\beta \subset D$ and $\bar{\gamma}_{r} \subset \psi_{1}(\beta)$. It can be shown that:

(22) $\beta$ contains a continuum $\beta^{\prime}$ such that $\psi_{1}\left(\beta^{\prime}\right)=\bar{\gamma}_{r}$. If $\beta^{\prime}$ is an arc whose endpoints are $e_{1}$ and $e_{2}$ then $\psi_{1}\left(\left\{e_{1}, e_{2}\right\}\right)=\left\{z_{1}, z_{2}\right\}$ and $\psi_{1}\left(\beta^{\prime} \backslash\left\{e_{1}, e_{2}\right\}\right)=\gamma_{r}$.

Further, we show:

(23) If $U$ is an open set contained in $D$ such that $E \cap \operatorname{Bd} U \neq \phi$, then $U \cap \psi(\beta \times I) \neq \phi$.

For $U$ meets a component $U_{n}$ of $\bar{B}_{n} \overline{B_{n+1}}$ for some $n$. By (14), (16), and 1.5, $U_{n}$ is an annulus. From (16), (17), (18), and 1.9, $U_{n}=$ $\psi\left(\beta \times\left[t_{n}, t_{n+1}\right]\right)$, and (23) follows.

Let $y_{0} \in \gamma_{r}$. By (23) there are continua $P_{k}(k=1,2)$ such that $\beta^{\prime}=P_{k}$ satisfies (22) and $P_{k} \cap\left(\operatorname{Int} X_{k}\right) \cap B\left(y_{0}, \varepsilon_{1}\right) \neq \phi$. It can be shown that $P_{1} \cap P_{2}=\phi$. By (17), for all $n$,

(24) $\psi\left(P_{1} \times\left\{t_{n}\right\}\right) \cap \psi\left(P_{2} \times\left\{t_{n}\right\}\right)=\phi$.

It can be shown that not both of $P_{1}$ and $P_{2}$ are simple closed curves. Hence we assume $P_{1}$ is an arc. Then $P_{2}$ is an arc or a simple closed curve. 
By (22) we may assume the endpoints $a_{1}^{1}$ and $b_{1}^{1}$ of $P_{1}$ satisfy $\psi_{1}\left(a_{1}^{1}\right)=z_{1}, \psi_{1}\left(b_{1}^{1}\right)=z_{2}$. If $P_{2}$ is an arc then we may assume its endpoints $a_{1}^{2}$ and $b_{1}^{2}$ satisfy $\psi_{1}\left(a_{1}^{2}\right)=z_{1}, \psi_{1}\left(b_{1}^{2}\right)=z_{2}$. If $P_{2}$ is a simple closed curve then $z_{1}=z_{2}$, and by analogy with the above we choose $a_{1}^{2}=b_{1}^{2} \in P_{2} \cap \psi_{1}^{-1}\left(z_{1}\right)$.

By (19), $\eta^{k}=\psi\left(\left\{a_{1}^{k}\right\} \times I\right)$ and $\xi^{k}=\psi^{\prime}\left(\left\{b_{1}^{k}\right\} \times I\right)$ are arcs. By (17) and (18) we have

(25) $\eta^{1} \backslash\left\{z_{1}\right\}, \eta^{2} \backslash\left\{z_{1}\right\}, \xi^{1} \backslash\left\{z_{2}\right\}$ (and $\xi^{2} \backslash\left\{z_{2}\right\}$ if $\xi^{2} \neq \eta^{2}$ ) are pairwise disjoint.

Let $p_{k} \in P_{k} \cap \psi_{1}^{-1}\left(y_{0}\right), k=1,2$. Let $P_{a}^{1}$ be the arc of $P_{1}$ from $a_{1}^{1}$ to $p_{1}$. Let $P_{b}^{1}$ be the arc of $P_{1}$ from $p_{1}$ to $b_{1}^{1}$. If $a_{1}^{2} \neq b_{1}^{2}$, let $P_{a}^{2}$ and $P_{b}^{2}$ be the arcs of $P_{2}$ from $a_{1}^{2}$ to $p_{2}$ and from $p_{2}$ to $b_{1}^{2}$, respectively. If $a_{1}^{2}=b_{1}^{2}$ then $z_{1}=z_{2}$. Then let $P_{a}^{2}$ be the arc of $p_{2}$ from $a_{1}^{2}$ to $p_{2}$ contained in $P_{2} \cap \psi_{1}^{-1}\left(\psi_{1}\left(P_{a}^{1}\right)\right)$ and let $P_{b}^{2}$ be the other arc of $P_{2}$ from $a_{1}^{2}$ to $p_{2}$.

Clearly $T_{1}=\bigcup_{k=1}^{2}\left[\eta^{k} \cup P_{a}^{k} \cup \psi\left(\left\{p_{k}\right\} \times I\right)\right]$ and $T_{2}=\bigcup_{k=1}^{2}\left[\xi^{k} \cup P_{b}^{k} \cup\right.$ $\left.\psi\left(\left\{p_{k}\right\} \times I\right)\right]$ are simple closed curves that are deformed by $\psi$ into proper subsets of $\alpha_{1}$. By 1.6, $T_{1}$ and $T_{2}$ bound disks $M_{1}$ and $M_{2}$ respectively in $B_{1}$. Clearly $M_{k}=\psi\left(T_{k} \times I\right)$.

There is an arc $\lambda_{1}^{\prime}$ in $M_{1} \cap B\left(z_{1}, \varepsilon_{1}\right)$ from $a_{1}^{1}$ to $a_{1}^{2}$ such that $\left\{a_{1}^{1}, a_{1}^{2}\right\}=$ $\lambda_{1}^{\prime} \cap \mathrm{Bd} M_{1}$. Then $\lambda_{1}^{\prime} \subset B_{1} \cap B\left(z_{1}, \varepsilon_{1}\right)$ and $\lambda_{1}^{\prime} \cap \mathrm{Bd} B_{1}=\left\{a_{1}^{1}, a_{1}^{2}\right\}$. By (19), $M_{1} \backslash\left\{z_{1}, y_{0}\right\}$ is a (noncompact) polyhedron, so by 1.7 there is an ambient isotopy of $M_{1}$ that is fixed on $\left(M_{1} \backslash B\left(z_{1}, \varepsilon_{1}\right)\right) \cup \mathrm{Bd} M_{1}$ and that carries $\lambda_{1}^{\prime}$ onto a polyhedral arc $\lambda_{1}$. Similarly, there is a polyhedral arc $\mu_{1}$ in $M_{2} \cap B\left(z_{2}, \varepsilon_{1}\right)$ from $b_{1}^{1}$ to $b_{1}^{2}$ such that $\left\{b_{1}^{1}, b_{1}^{2}\right\}=\mu_{1} \cap \mathrm{Bd} B_{1}$.

For all $n$, let $a_{n}^{k}=\psi\left(a_{1}^{k}, t_{n}\right) \in \mathrm{Bd} B_{n}$, and let $b_{n}^{k}=\psi\left(b_{1}^{k}, t_{n}\right) \in \operatorname{Bd} B_{n}$. Let $\eta_{0}^{k}=\eta^{k}, \xi_{0}^{k}=\xi^{k}, \eta_{n}^{k}=\psi\left(\left\{a_{n}^{k}\right\} \times\left[t_{n+1}, 1\right]\right.$ ) (the arc of $\eta^{k}$ from $a_{n+1}^{k}$ to $\left.z_{1}\right), \xi_{n}^{k}=\psi\left(\left\{b_{n}^{k}\right\} \times\left[t_{n+1}, 1\right]\right)$ (the arc of $\xi^{k}$ from $b_{n+1}^{k}$ to $z_{2}$ ). Note that we have begun an induction argument by showing that for $n=1$, the following statements (26) through (29) are valid:

(26) There are polyhedral arcs $\lambda_{n} \subset M_{1} \cap B_{n} \cap B\left(z_{1}, \varepsilon_{n}\right)$ from $a_{n}^{1}$ to $a_{n}^{2}, \mu_{n} \subset M_{2} \cap B_{n} \cap B\left(z_{2}, \varepsilon_{n}\right)$ from $b_{n}^{1}$ to $b_{n}^{2}$ such that:

(27) $\left\{a_{n}^{1}, a_{n}^{2}\right\}=\lambda_{n} \cap \mathrm{Bd} B_{n}=\lambda_{n} \cap \mathrm{Bd} M_{1}$.

$$
\left\{b_{n}^{1}, b_{n}^{2}\right\}=\mu_{n} \cap \mathrm{Bd} B_{n}=\mu_{n} \cap \mathrm{Bd} M_{2} .
$$

(28) $\quad \lambda_{n} \cap\left(\eta_{n}^{1} \cup \eta_{n}^{2}\right)=\dot{\phi}=\mu_{n} \cap\left(\xi_{n}^{1} \cup \xi_{n}^{2}\right)$.

(For $n=1,(27)$ and (28) follow from observing which points are left fixed by the ambient isotopies.)

(29) $\lambda_{n} \cap \lambda_{j}=\phi=\mu_{n} \cap \mu_{j}$ for $j<n$.

Suppose $m>0$ and (26) through (29) are valid for $n=1, \cdots, m$. The inductive step is done as above, with obvious modifications. For example, to obtain $\lambda_{m+1}$ satisfying (26) through (29), we work in the disk bounded not by $T_{1}$, but by the simple closed curve

$$
\overline{u_{m} v_{m}} \cup \overline{u_{m} a_{m+1}^{1}} \cup \eta_{m}^{1} \cup \eta_{m}^{2} \cup \overline{v_{m} a_{m+1}^{2}},
$$


where $\overline{u_{m} v_{m}}$ is the arc of $\lambda_{m}$ whose endpoints $u_{m}$ and $v_{m}$ satisfy $u_{m} \in \psi\left(P_{1} \times\left\{t_{m}\right\}\right), v_{m} \in \psi\left(P_{2} \times\left\{t_{m}\right\}\right), \overline{u_{m} v_{m}} \backslash\left\{u_{m}, v_{m}\right\} \subset \operatorname{Int} B_{m+1} ; \overline{u_{m} a_{m+1}^{1}}$ is the arc of $\psi\left(P_{1} \times\left\{t_{m+1}\right\}\right)$ from $u_{m}$ to $a_{m+1}^{1}$; and $\overline{v_{m} a_{m+1}^{2}}$ is the arc of $M_{1} \cap \psi\left(P_{2} \times\left\{t_{m+1}\right\}\right)$ from $v_{m}$ to $a_{m+1}^{2}$. Thus (26) through (29) hold for all $n$.

Since $\lambda_{n} \subset M_{1}, \mu_{n} \subset M_{2}$, and $\left(\mathrm{Bd} M_{1}\right) \cap\left(\mathrm{Bd} M_{2}\right) \backslash \psi\left(\left\{p_{1}, p_{2}\right\} \times I\right)=$ $\eta^{2} \cap \xi^{2},(25)$ and (27) imply

$$
\lambda_{n} \cap \mu_{j}=\left\{\begin{array}{l}
\phi \text { if } n \neq j, \text { or if } n=j \text { and } \eta^{2} \neq \xi^{2} \\
\left\{a_{n}^{2}=b_{n}^{2}\right\} \text { if } n=j \text { and } \eta^{2}=\xi^{2} .
\end{array}\right.
$$

For $k=1,2$, let $Q_{k}$ be the boundary curve of $B_{1}$ containing $P_{k}$. Let $Q_{l_{t}}^{n}=\psi\left(Q_{k} \times\left\{t_{n}\right\}\right), P_{k}^{n}=\psi\left(P_{k} \times\left\{t_{n}\right\}\right)$. Let $E_{n}=\left[\left(Q_{1}^{n} \cup Q_{2}^{n}\right) \backslash\left(P_{1}^{n} \cup P_{2}^{n}\right)\right] \cup$ $\lambda_{n} \cup \mu_{n}$. Clearly $E_{n}$ is a polyhedron, and $E_{n} \cap E_{j}=\phi$ for $n \neq j$. If $Q_{1} \neq Q_{2}$, then (17), (24), (27), and (30) imply $E_{n}$ is a simple closed curve. (Note (30) implies if $\lambda_{n} \cap \mu_{n}=\left\{a_{n}^{2}\right\}$ then $Q_{2}^{n}=P_{2}^{n}$, so $E_{n}=\left(Q_{1}^{n} \backslash P_{1}^{n}\right) \cup \lambda_{n} \cup \mu_{n}$.) Similarly, if $Q_{1}=Q_{2}$ then either $E_{n}$ is a simple closed curve for all $n$ or $E_{n}$ is a disjoint union of two simple closed curves for all $n$.

For all $n$, let $J_{n} \subset M_{1}$ be the disk bounded by $\eta_{n-1}^{1} \cup \eta_{n-1}^{2} \cup \lambda_{n}$ and let $J_{n}^{\prime} \subset M_{2}$ be the disk bounded by $\xi_{n-1}^{1} \cup \xi_{n-1}^{2} \cup \mu_{n}$. Define $A_{n}=$ $\left[B_{n} \backslash\left(M_{1} \cup M_{2}\right)\right] \cup J_{n} \cup J_{n}^{\prime}$. To complete the proof, we must show (13) through (20) are satisfied when $\left(\left\{A_{n}\right\}_{n=1}^{\infty}, C\right)$ replaces $\left(\left\{B_{n}\right\}_{n=1}^{\infty}, C^{\prime}\right)$ and an appropriate map $h$ replaces $\psi$.

We have

$$
\mathrm{Bd} A_{n}=E_{n} \cup\left[\left(\mathrm{Bd} B_{n}\right) \backslash\left(Q_{1}^{n} \cup Q_{2}^{n}\right)\right] \text { and } E_{n} \cap\left[\left(\operatorname{Bd} B_{n}\right) \backslash\left(Q_{1}^{n} \cup Q_{2}^{n}\right)\right]=\dot{\phi} \text {. }
$$

Therefore $A_{n}$ is a polyhedral bounded surface. The analogue of (13) is satisfied.

Since $E_{n} \cap E_{j}=\phi$ for $n \neq j,\left(\operatorname{Bd} A_{n}\right) \cap\left(\operatorname{Bd} A_{j}\right)=\phi$. Clearly $z_{1} \in$ $J_{n+1} \subset J_{n}$ and $z_{2} \in J_{n+1}^{\prime} \subset J_{n}^{\prime}$. It follows that $C \subset A_{n+1} \subset \operatorname{Int} A_{n}$. The analogue of (14) is satisfied.

It is easily seen that there are maps $h^{\prime}: J_{1} \times I \rightarrow J_{1}$ and $h^{\prime \prime}: J_{1}^{\prime} \times$ $I \rightarrow J_{1}^{\prime}$ such that for all $x \in \eta^{1} \cup \eta^{2}, y \in \xi^{1} \cup \xi^{2}, t \in I$,

(31) $h^{\prime}(x, t)=\psi(x, t) ; h^{\prime \prime}(y, t)=\psi(y, t)$; and such that $h^{\prime}$ and $h^{\prime \prime}$ satisfy analogues of (15) through (19):

$\left(15^{\prime}\right) \quad h^{\prime}$ is a strong deformation retraction of $J_{1}$ onto $\left\{z_{1}\right\}$, and for all $n$ :

(16') $h^{\prime} \mid J_{n} \times\left[t_{n}, t_{n+1}\right]$ is a strong deformation retraction of $J_{n}$ onto $J_{n+1}$.

(17') $h^{\prime} \mid \lambda_{n} \times\left[t_{n}, t_{n+1}\right]$ is an isotopy of $\lambda_{n}$ onto $\lambda_{n+1}$. 
(18') If $x \in h^{\prime}\left(\{y\} \times\left[t_{n}, t_{n+1}\right]\right)$ for $y \in \lambda_{n}$, then $h^{\prime}\left(\{x\} \times\left[t_{n}, t_{n+1}\right]\right) \subset h^{\prime}(\{y\} \times$ $\left.\left[t_{n}, t_{n+1}\right]\right)$ and $h^{\prime}(x, t)=h^{\prime}(y, t)$ for $t \in\left[t_{n+1}, 1\right]$.

$\left(19^{\prime}\right)$ For all $x \in \lambda_{n}, h^{\prime}(\{x\} \times I)$ is an arc and $h^{\prime}(\{x\} \times[0,1))$ is a (noncompact) polyhedron.

Similar versions of $\left(15^{\prime}\right)$ through $\left(19^{\prime}\right)$ hold upon replacing $\left(h^{\prime},\left\{J_{n}\right\}_{n=1}^{\infty}, z_{1},\left\{\lambda_{n}\right\}_{n=1}^{\infty}\right)$ by $\left(h^{\prime \prime},\left\{J_{n}^{\prime}\right\}_{n=1}^{\infty}, z_{2},\left\{\mu_{n}\right\}_{n=1}^{\infty}\right)$.

Define a map $h$ on $A_{1} \times I$ by

$$
h(x, t)=\left\{\begin{array}{lll}
h^{\prime}(x, t) & \text { if } & x \in J_{1} \\
h^{\prime \prime}(x, t) & \text { if } & x \in J_{1}^{\prime} \\
\psi(x, t) & \text { otherwise }
\end{array}\right.
$$

By (31), $h$ is well-defined and continuous. From (17) and (18),

(32) if $x \in B_{n} \backslash\left(M_{1} \cup M_{2}\right)$ then $\psi(\{x\} \times I) \subset B_{n} \backslash\left(M_{1} \cup M_{2} \backslash\left\{z_{1}, z_{2}\right\}\right)$.

By (15), $\left(15^{\prime}\right)$, and (32), $h\left(A_{1} \times I\right)=A_{1}$. Clearly $h(x, t)=x$ for all $(x, t) \in C \times I$, and $h_{1}\left(A_{1}\right)=C$. Thus $h$ satisfies the analogue of (15).

For all $n$ :

By (16), $\left(16^{\prime}\right)$, and (32), $h$ satisfies the analogue of (16).

By (17), (17'), and (32), $h$ satisfies the analogue of (17).

By (18) and (18'), $h$ satisfies the analogue of (18).

By (19) and $\left(19^{\prime}\right), h$ satisfies the analogue of (19).

By (20) and our construction of $E_{n}, h$ satisfies the analogue of (20). The proof of Theorem 2.5 is completed.

3. Arcs. Let $X$ be a finite-dimensional compactum and let $\left\{C_{0}, C_{1}\right\} \subset 2_{h}^{X}$. Under what circumstances is there an arc in $2_{h}^{X}$ from $C_{0}$ to $C_{1}$ ? In [1], it was found that a necessary but insufficient condition is that $C_{0}$ and $C_{1}$ have the same homotopy type; and a sufficient but unnecessary condition is that $C_{0}$ and $C_{1}$ be isotopic in $X$. For $X=M$, we obtain a condition that is both necessary and sufficient:

THEOREM 3.1. Let $\left\{C_{0}, C_{1}\right\} \subset 2_{h}^{M} \backslash\{M\}$. By 2.5, there exist $A_{j} \in$ $2_{h}^{M}(j=0,1)$ such that each component of $A_{j}$ is a bounded surface, $C_{j} \subset \operatorname{Int} A_{j}$, and $C_{j}$ is a strong deformation retract of $A_{j}$. Then there is an arc in $2_{h}^{M}$ from $C_{0}$ to $C_{1}$ if and only if there is an ambient isotopy of $M$ taking $A_{0}$ onto $A_{1}$.

First we prove:

Lemma 3.2. Suppose $C \in 2_{h}^{M} \backslash\{M\}$, and let $\left\{A_{n}\right\}_{n=1}^{\infty},\left\{t_{n}\right\}_{n=1}^{\infty}$, and $h$ be as in 2.5. Then there is an arc $\mathscr{A}$ in $2_{h}^{M}$ from $A_{1}$ to $C$ containing 
each $A_{n}$ such that if $A \in \mathscr{A} \backslash\{C\}$, each component of $A$ is a bounded surface.

Proof. Recall the notation in the statement of Theorem 2.5. In the proof of 2.5, we saw:

(1) $h \mid\left(\mathrm{Bd} A_{n}\right) \times\left[t_{n}, t_{n+1}\right]$ is an isotopy of $\mathrm{Bd} A_{n}$ onto $\mathrm{Bd} A_{n+1}$.

It follows from (16) and (18) of the proof of 2.5 that

(2) if $x \in \mathrm{Bd} A_{n}$ then $h\left(\{x\} \times\left[t_{n}, t_{n+1}\right]\right)=\gamma_{x}$ is an arc such that $\gamma_{x} \backslash\left\{x, h\left(x, t_{n+1}\right)\right\} \subset\left(\operatorname{Int} A_{n}\right) \backslash A_{n+1}$.

If $\varepsilon_{n}=\sup \left\{\operatorname{diam} h(\{x\} \times I) \mid x \in A_{n}\right\}$, then $\lim \varepsilon_{n}=0$, and by 1.1, $A_{n} \underset{\rho_{h}}{\rightarrow} C$, so it follows that there is a sequence of positive numbers $\delta_{n}$ such that

(3) $\lim \delta_{n}=0$, and for all $n, s\left(A_{n}, 6 \varepsilon_{n}, \delta_{n}\right)$.

Let $P$ be a component of $\overline{A_{n} \backslash A_{n+1}}$ By $2.5(\mathrm{a}), 2.5(\mathrm{~b}), 2.5(\mathrm{~d})$, and 1.5, $P$ is an annulus. Let the boundary curves of $P$ be $\alpha_{n} \subset \mathrm{Bd} A_{n}$ and $\alpha_{n+1} \subset \mathrm{Bd} A_{n+1}$. There is a set $E=\left\{x_{0}, x_{1}, \cdots, x_{k-1}\right\} \subset \alpha_{n}$ of $k$ distinct points numbered according to an orientation of $\alpha_{n}$ (let $x_{k}=x_{0}$ ) such that if $\beta_{j}$ is the arc of $\alpha_{n}$ from $x_{j-1}$ to $x_{j}$ containing no other member of $E$, then $\operatorname{diam} \beta_{j}<\varepsilon_{n}$. For each $j$, let $y_{j}=h\left(x_{j}, t_{n+1}\right)$. By (2), $\gamma_{j}=h\left(\left\{x_{j}\right\} \times\left[t_{n}, t_{n+1}\right]\right)$ is an arc from $x_{j}$ to $y_{j}$ such that $\gamma_{j} \backslash\left\{x_{j}, y_{j}\right\} \subset \operatorname{Int} P$. By (1), the $\gamma_{j}$ are pairwise disjoint for $j \in$ $\{0,1, \cdots, k-1\}\left(\gamma_{k}=\gamma_{0}\right)$ and (also by $\left.(1)\right) \zeta_{j}=h\left(\beta_{j} \times\left\{t_{n+1}\right\}\right)$ is an arc of $\alpha_{n+1}$ from $y_{j-1}$ to $y_{j}$ not containing $y_{m}$ if $y_{m} \notin\left\{y_{j-1}, y_{j}\right\}$. Clearly $\operatorname{diam} \gamma_{j} \leqq \varepsilon_{n}$.

Let $\left\{y, y^{\prime}\right\} \subset \zeta_{j}$. There exist $x, x^{\prime} \in \beta_{j}$ such that $y=h\left(x, t_{n+1}\right)$ and $y^{\prime}=h\left(x^{\prime}, t_{n+1}\right)$. Then $\rho\left(y, y^{\prime}\right) \leqq \rho(y, x)+\rho\left(x, x^{\prime}\right)+\rho\left(x^{\prime}, y^{\prime}\right) \leqq \varepsilon_{n}+$ $\operatorname{diam} \beta_{j}+\varepsilon_{n}<3 \varepsilon_{n}$. Therefore $\operatorname{diam} \zeta_{j}<3 \varepsilon_{n}$.

Let $S_{j}$ be the simple closed curve in $P$ defined by $S_{j}=\gamma_{j-1} \cup$ $\beta_{j} \cup \gamma_{j} \cup \zeta_{j}$. Then $\operatorname{diam} S_{j} \leqq \operatorname{diam} \gamma_{j-1}+\operatorname{diam} \beta_{j}+\operatorname{diam} \gamma_{j}+\operatorname{diam} \zeta_{j}<$ $\varepsilon_{n}+\varepsilon_{n}+\varepsilon_{n}+3 \varepsilon_{n}=6 \varepsilon_{n}$. By (3) and 1.6, $S_{j}$ bounds a disk $K_{j} \subset A_{n}$ such that

(4) $\operatorname{diam} K_{j}<\delta_{n}$.

Indeed $K_{j} \subset P$, for if $K_{j}^{\prime}$ is the disk in $P$ bounded by $S_{j}$ and $K_{j}^{\prime} \neq K_{j}$, then $K_{j} \cap K_{j}^{\prime}=S_{j}$ and $K_{j} \cup K_{j}^{\prime}$ is a 2 -sphere in $A_{n}$, which is impossible.

It is easily seen that there is a map $F: P \times I \rightarrow P$ that is a strongly contracting strong deformation retraction and a pseudoisotopy of $P$ to $\alpha_{n+1}$ such that $F\left(K_{j} \times I\right) \subset K_{j}$ for all $j$. From (4) we have

(5) $F_{t}$ is a $\delta_{n}$-embedding for $0 \leqq t<1$.

Apply the above construction to each component of $\overline{A_{n} \backslash A_{n+1}}$. In the above, $F_{t} \mid \alpha_{n+1}=\operatorname{Id}_{\alpha_{n+1}}$ for all $t \in I$, so we may extend each $F_{t}$ via the identity to obtain a map $F^{n}: A_{n} \times I \rightarrow A_{n}$ that is a strongly contracting strong deformation retraction and a pseudoisotopy of 
$A_{n}$ onto $A_{n+1}$ moving no point by as much as $\delta_{n}$. Let $a_{n}: I \rightarrow 2_{h}^{\mu}$ be defined by $a_{n}(t)=F^{n}\left(A_{n} \times\{t\}\right)$. By $1.3, a_{n}$ is continuous for $0 \leqq t<1$. By 1.1, $a_{n}$ is continuous for $t=1$.

Let $L: I \rightarrow 2_{h}^{M}$ be defined by

$$
L(t)= \begin{cases}a_{n}\left[\frac{t-t_{n}}{t_{n+1}-t_{n}}\right] & \text { if } \quad t_{n} \leqq t \leqq t_{n+1} ; \\ C & \text { if } t=1\end{cases}
$$

Since $a_{n}(1)=A_{n+1}=a_{n+1}(0), L$ is well-defined; and $L$ is continuous for $0 \leqq t<1$. From (3), (5), and 1.2, $L$ is continuous for $t=1$. Since $L(0)=A_{1}$ and $L(1)=C, L(I)$ contains an arc in $2_{h}^{M}$ from $A_{1}$ to $C$. The second conclusion of the lemma follows from the fact that for all $n, F^{n}$ is a pseudoisotopy of $A_{n}$ onto $A_{n+1}$.

We show the existence of a basis with useful properties.

Lemma 3.3. Let $C \in 2_{h}^{M} \backslash\{M\}$ and let $\varepsilon>0$. By 1.1 and 2.5, there exists $A$ such that $\rho_{h}(A, C)<\varepsilon$, each component of $A$ is a bounded surface, $C \subset \operatorname{Int} A$, and $C$ is a strong deformation retract of $A$. There is a neighborhood $\mathscr{W}$ of $C$ in $2_{h}^{M}$ such that $X \in \mathscr{C}$ implies $\rho_{h}(X, C)<\varepsilon, X \subset \operatorname{Int} A$, and $X$ is a strong deformation retract of A. Further, if each component of $X \in \mathscr{C}$ is a bounded surface, then there is an ambient isotopy of $M$ that carries $A$ onto $X$.

Proof. We may assume $A$ is a polyhedron, and that $\varepsilon$ is so small that two maps $f_{0}, f_{1}: C \rightarrow A$ such that $\rho\left(f_{0}, f_{1}\right)<\varepsilon$ are homotopic in $A$. Recall $[C]_{M}=\left\{X \in 2_{h}^{H} \mid X\right.$ and $C$ have the same homotopy type $\}$ is open. From 2.1 it follows that

$$
\mathscr{C}=[C]_{M} \cap\left\{X \in 2_{h}^{M} \mid X \subset \operatorname{Int} A\right\} \cap\left\{X \in 2_{h}^{M} \mid \rho_{h}(X, C)<\varepsilon\right\}
$$

is an open set in $2_{h}^{M}$ containing $C$.

We may assume $C$ and $A$ are connected (otherwise we apply the following by components). Let $X \in \mathscr{W}$. There is an $\varepsilon$-map $g: C \rightarrow X$. Let $i: C \rightarrow A, j: X \rightarrow A$ be inclusion maps. By choice of $\varepsilon, i_{*}=j_{*} \circ g_{*}$ : $\Pi_{1} C \rightarrow \Pi_{1} A$. By choice of $A, i_{*}$ is an isomorphism. Therefore $j_{*}: \Pi_{1} X \rightarrow \Pi_{1} A$ is a surjective homomorphism. But $\{X, A\} \subset[C]_{M}$, so $\Pi_{1} X$ and $\Pi_{1} A$ are isomorphic. Since $A$ is a bounded surface, $\Pi_{1} A$ is a finitely generated free group. Therefore $j_{*}$ is an isomorphism (see [10], p. 59).

Recall the definition of $\Delta X$ given in $\S 1$. Since $X$ and $A$ have the same homotopy type, $\Delta X=\Delta A$. But $\Delta A \leqq 1$, since if $A$ is a disk it has the homotopy of a point, while otherwise $A$ has the homotopy type of a wedge of finitely many simple closed curves. With $N=\Delta A \leqq 1$, we apply Whitehead's theorem ([12], 1, p. 1133) 
and conclude $j: X \rightarrow A$ is a homotopy equivalence.

By 1.1 and 2.5 there is a polyhedral bounded surface $B \in \mathscr{C}$ such that $X \subset$ Int $B$ and $X$ is a strong deformation retract of $B$. Applying the above to $B$, we conclude the inclusion of $B$ into $A$ is a homotopy equivalence. Hence $B$ is a strong deformation retract of $A$ (see [6], 3.2, p. 6). Thus $X$ is a strong deformation retract of $A$.

If $X \in \mathscr{Q}$ is a bounded surface, then by 1.5 each component of $\overline{A \backslash X}$ is an annulus. Let $S$ be a component of $\operatorname{Bd} A$. Let $A^{\prime}$ be the component of $\overline{A \backslash X}$ containing $S$. Let $S^{\prime}$ be the component of $\operatorname{Bd} A^{\prime}$ that lies in $X$. There are annuli $A_{1}$ and $A_{2}$ that collar $S$ in $\overline{M \backslash A}$ and $S^{\prime}$ in $X$ respectively. Then $A^{\prime \prime}=A_{1} \cup A^{\prime} \cup A_{2}$ is an annulus. There is an isotopy $h: A^{\prime \prime} \times I \rightarrow A^{\prime \prime}$ of $A^{\prime \prime}$ onto itself such that $h_{1}\left(A^{\prime} \cup A_{2}\right)=A_{2}, h_{1}\left(A_{1}\right)=A^{\prime} \cup A_{1}$, and $h(z, t)=z$ for $\operatorname{all}(z, t) \in\left(\operatorname{Bd} A^{\prime \prime}\right) \times I$. Apply this construction to each component of $\overline{A \backslash X}$ and extend via the identity on $M \backslash(\overline{A \backslash X})$ to get an ambient isotopy of $M$ that carries $A$ onto $X$.

Proof of Theorem 3.1. Suppose there is an ambient isotopy of $M$ taking $A_{0}$ onto $A_{1}$. By 1.3 , there is an arc in $2_{h}^{M}$ from $A_{0}$ to $A_{1}$. By 3.2, there are arcs in $2_{h}^{M}$ from $A_{0}$ to $C_{0}$ and from $A_{1}$ to $C_{1}$. Hence there is an arc in $2_{h}^{M}$ from $C_{0}$ to $C_{1}$.

Conversely, suppose there is an embedding $p: I \rightarrow 2_{h}^{M}$ such that $p(0)=C_{0}$ and $p(1)=C_{1}$. Since $p(I)$ is compact, 3.3 implies that there exist $0 \leqq t_{0}<t_{1}<\cdots<t_{m} \leqq 1 ; A_{t_{n}} \in 2_{h}^{M}$ such that each component of $A_{t_{n}}$ is a bounded surface; and neighborhoods $\mathscr{C}_{n}$ of $p\left(t_{n}\right)$ in $2_{k}^{M}$ such that if $X \in \mathscr{U}_{n}$ and each component of $X$ is a bounded surface then there is an ambient isotopy of $M$ taking $A_{t_{n}}$ onto $X$, and such that $\mathscr{U}_{n} \cap \mathscr{U}_{n+1} \neq \phi$ and $p(I) \subset \bigcup_{n=0}^{m} \mathscr{C}_{n}$. Further, 3.3 enables us to assume that $A_{0}=A_{t_{0}}$ and $A_{1}=A_{t_{m}}$.

By 1.1 and 2.5, for each $n<m$ there exists $B_{n} \in \mathscr{L}_{n} \cap \mathscr{L}_{n+1}$ such that each component of $B_{n}$ is a bounded surface. There are ambient isotopies of $M$ taking $A_{t_{n}}$ and $A_{t_{n+1}}$ onto $B_{n}$. Therefore there is an ambient isotopy of $M$ taking $A_{t_{n}}$ onto $A_{t_{n+1}}$. Hence there is an ambient isotopy of $M$ taking $A_{0}=A_{t_{0}}$ onto $A_{t_{m}}=A_{1}$.

4. Global properties. The spaces $D(N)$ and $L(N)$ of deformation retracts (respectively, compact AR subsets) of a compact 2-manifold $N$ were studied by Wagner in [11]. The topologies of these spaces may be described thus: $A_{n} \underset{D(N)}{\longrightarrow} C\left(A_{n} \underset{L(N)}{\longrightarrow} C\right)$ if and only if there are maps $r_{0}: N \rightarrow N, r_{n}: N \rightarrow N$ that are deformation retractions (that are retractions) of $N$ onto $C$ and $A_{n}$ respectively such that $r_{n} \rightarrow r_{0}$ uniformly on $N$. We show these spaces are closely related to $2_{h}^{M}$. 
We will need the following lemma. In both its statement and its proof, it is similar to ([2], 3.1, pp. 212-213).

Lemma 4.1. If $C \in 2_{h}^{M} \backslash\{M\}, C$ is connected, and $\varepsilon>0$, there is $a \delta>0$ and $a$ neighborhood $\mathscr{U}$ of $C$ in $2_{h}^{M}$ such that if $\{A, B\} \subset \mathscr{C}$, $B \subset A$, and $A$ is a bounded surface, then every pair of points in $\mathrm{Bd} A$ that can be joined by a $\delta$-arc in $M \backslash B$ can be joined by an $\varepsilon$ arc in $\mathrm{Bd} A$.

Proof. By 3.3, there is a neighborhood $\mathscr{C}_{1}$ of $C$ in $2_{h}^{M}$ and a bounded surface $N \subset M$ such that for all $X \in \mathscr{C}_{1}$ we have $X \subset \operatorname{Int} N$ and $X$ is a strong deformation retract of $N$.

Since $M$ is an ANR, there exists $\eta>0$ such that $s(M, \eta, \varepsilon / 4)$. Also there is a $\delta>0$ such that:

(1) If $N$ has more than one boundary curve then

$\delta<\min \{\rho(S, T) \mid S$ and $T$ are distinct boundary curves of $N\}$.

(2) $\delta<1 / 2 \min \{\eta, \varepsilon\}$.

(3) There is a neighborhood $\mathscr{U}_{2}$ of $C$ in $2_{h}^{M}$ such that if $X \in \mathscr{Z}_{2}$ then $s(X, \delta, \eta / 2)$.

Let $\mathscr{C}_{3}=\left\{X \in 2_{h}^{M} \mid \rho_{h}(X, C)<\delta / 2\right\}$. Let $\mathscr{C}_{C}=\mathscr{C}_{1} \cap \mathscr{C}_{2} \cap \mathscr{C}_{3}$. Clearly $\mathscr{C}$ is a neighborhood of $C$ in $2_{h}^{M}$.

Suppose $\{A, B\} \subset \mathscr{U}$ such that $B \subset A$ and $A$ is a bounded surface. From 1.4 (with $R=B$ ) it follows that $B$ separates each pair of boundary curves of $N$ in $N$. Since each component of $\overline{N \backslash A}$ is an annulus, it follows that

(4) $B$ separates each pair of distinct boundary curves of $A$ in $A$.

Let $p$ and $q$ be distinct points of $\mathrm{Bd} A$ such that there is a $\delta$-arc $\beta$ from $p$ to $q$ in $M \backslash B$.

Suppose $\beta$ meets distinct boundary curves $T_{1}$ and $T_{2}$ of $A$. It follows from (4) that $\beta$ must contain a $\delta$-arc $\beta^{\prime}$ from $p^{\prime} \in T_{1}$ to $q^{\prime} \in T_{2}$ such that $\beta^{\prime} \cap A=\left\{p^{\prime}, q^{\prime}\right\}$. For $n=1,2$, let $B_{n}$ be the annular component of $\overline{N \backslash A}$ containing $T_{n}$ and let $T_{n}^{\prime}$ be the component of $\operatorname{Bd} N$ that is contained in $B_{n}$. By $1.4, T_{1}^{\prime} \neq T_{2}^{\prime}$. By (4) and 1.4, there are distinct components $B_{n}^{\prime}$ of $N \backslash B$ such that Int $B_{n} \subset B_{n}^{\prime}$. Then $T_{n} \subset B_{n} \subset \overline{B_{n}^{\prime}}$, so we must have $\beta^{\prime} \cap \mathrm{Bd} B_{n}^{\prime} \neq \phi$. Since $\mathrm{Bd} B_{n}^{\prime} \subset T_{n}^{\prime} \cup$ $\mathrm{Bd} B$ and $\beta^{\prime} \cap \mathrm{Bd} B \subset \beta^{\prime} \cap B=\phi$, we have $\beta^{\prime} \cap T_{n}^{\prime} \neq \phi$ for $n=1,2$. The latter contradicts (1). We conclude that $\beta \cap \mathrm{Bd} A$ is contained in a single component $J$ of $\mathrm{Bd} A$.

By $N_{s}(\beta)$ we will mean the set of all points in $M$ whose distance from $\beta$ is less than $s$. Since $\operatorname{diam} \beta<\delta$, there is an $s>0$ such that $\operatorname{diam} N_{s}(\beta)<\delta$. By the proof of 2.4, we may assume $\beta \cap J$ has finitely many components. If $\gamma$ is a component of $\beta \cap J$ 
that is not a single point, then $\gamma$ is an arc with endpoints $b, c$. There is an arc $\gamma^{\prime} \subset N_{s}(\beta) \backslash B$ from $b$ to $c$ such that $\gamma^{\prime} \cap J=\{b, c\}$. If $\gamma_{1}, \cdots, \gamma_{m}$ are the components of $\beta \cap J$ that are arcs, then $\beta_{1}=$ $\left(\beta \backslash \bigcup_{n=1}^{n} \gamma_{n}\right) \cup \bigcup_{n=1}^{m} \gamma_{n}^{\prime}$ meets $J$ in but finitely many points and (by choice of $s$ ) contains a $\delta$-arc $\beta_{2}$ from $p$ to $q$. Thus (by replacing $\beta$ by $\beta_{2}$ if necessary) we may assume $\beta \cap J$ is a finite set.

Suppose $\beta \cap J=\{p, q\}$. We consider two cases:

( I ) Suppose $\beta \backslash\{p, q\} \subset M \backslash A$. Since $\operatorname{diam} \beta<\delta$, (3) implies there is an $\eta / 2$-arc $\xi$ in $A$ from $p$ to $q$. We assume $\xi \backslash\{p, q\} \subset \operatorname{Int} A$. Then $K=\beta \cup \xi$ is a simple closed curve and diam $K<\delta+\eta / 2<\eta$ (by (2)). By 1.6 and our choice of $\eta, K$ bounds a disk $L \subset M$ with diam $L<\varepsilon / 4$.

Let $x \in \beta \backslash\{p, q\}, y \in \xi \backslash\{p, q\}$. For any fixed $r>0, B(x, r) \cap(M \backslash A) \neq$ $\phi \neq B(y, r) \cap \operatorname{Int} A$. Suppose $L$ fails to contain an arc of $J$ from $p$ to $q$. Our choices of $\beta$ and $\xi$ imply $J \cap K=J \cap \operatorname{Bd} L=\{p, q\}$, so the assumption implies $J \cap L=\{p, q\}$. Thus $\phi=J \cap \operatorname{Int} L=(\operatorname{Bd} A) \cap \operatorname{Int} L$. Since $\phi \neq B(y, r) \cap \operatorname{Int} A$ meets Int $L \cap \operatorname{Int} A$ and $\dot{\phi} \neq B(x, r) \cap(M \backslash A)$ meets Int $L \cap(M \backslash A)$, it follows that $\operatorname{Int} L=(\operatorname{Int} L \cap \operatorname{Int} A) \cup$ (Int $L \cap(M \backslash A)$ ) is disconnected. This is impossible, so $L$ contains an arc of $J$ from $p$ to $q$ that lies in $N_{\varepsilon / 4}(\beta)$ (since $\beta \subset L$ and $\operatorname{diam} L<\varepsilon / 4)$.

(II) Suppose $\beta \backslash\{p, q\} \subset \operatorname{Int} A$. Then $A=A_{1} \cup A_{2}$, where $A_{1}$ is a bounded surface containing $B, A_{2}$ is (by (4) and the fact that $\beta \subset M \backslash B)$ a bounded surface whose boundary is the union of $\beta$ and an arc of $J$ from $p$ to $q$, and $A_{1} \cap A_{2}=\beta$. By choice of $\mathscr{C}_{3}$, there is a $\delta$-map $f: A \rightarrow B$. If $z \in A_{2}$ then $f(z) \in B \subset A_{1}$, so by (3) there is an $\eta / 2$-arc $\zeta \subset A$ from $z$ to $f(z)$. Clearly $\zeta$ meets $\beta$. Hence $A_{2} \subset$ $N_{\eta / 2}(\beta)$. In particular, the arc of $J$ from $p$ to $q$ that lies in $\mathrm{Bd} A_{2}$ must lie in $N_{\eta / 2}(\beta)$.

Our choice of $\eta$ implies $\eta / 2<\varepsilon / 4$. In both (I) and (II), $J$ contains an arc from $p$ to $q$ that lies in $N_{\varepsilon / 4}(\beta)$.

More generally, if $\beta \cap J=\left\{p=p_{1}, \cdots, p_{k}=q\right\}$ where the $p_{n}$ are numbered in order from $p$ to $q$ along $\beta$, then each subarc $\overline{p_{n} p_{n+1}}$ of $\beta$ satisfies the condition of (I) or (II). For each $n<k$ there is an arc $\zeta_{n}$ of $J$ from $p_{n}$ to $p_{n+1}$ in $N_{\varepsilon / 4}(\beta)$. There is an $\operatorname{arc} \zeta_{0} \subset \bigcup_{n=1}^{k-1} \zeta_{n} \subset N_{\varepsilon / 4}(\beta)$ of $J$ from $p$ to $q$. Observe $\operatorname{diam} \zeta_{0} \leqq \operatorname{diam} N_{\varepsilon / 4}(\beta) \leqq \varepsilon / 2+\operatorname{diam} \beta<$ $\varepsilon / 2+\delta<\varepsilon$ (by (2)).

We now strengthen 3.3.

Lemma 4.2. Let $C \in 2_{h}^{M} \backslash\{M\}, \varepsilon>0$. Then there exist $N \in 2_{h}^{M}$ and a neighborhood $\mathscr{C}$ of $C$ in $2_{h}^{M}$ such that each component of $N$ is a bounded surface and such that for all $X \in \mathscr{W}, \rho_{h}(X, C)<\varepsilon, X \subset \operatorname{Int} N$, and there is a strong deformation retraction $h: N \times I \rightarrow N$ of $N$ onto $X$ such that for each $t \in I, h_{t}$ is an $\varepsilon-m a p$. 
Proof. It follows from ([2], 2.1, p. 210) that there is no loss of generality in assuming $C$ is connected.

There is a neighborhood $\mathscr{U}_{1}$ of $C$ in $2_{h}^{M}$ and a $\delta>0$ such that

(1) if $X \in \mathscr{Q}_{1}$ then $s(X, \delta, \varepsilon / 2)$.

There are positive numbers $\delta_{1}$ and $\delta_{2}$ such that

(2) $17 \delta_{1}+\delta_{2}<\delta$

and (by 4.1) such that

(3) there is a neighborhood $\mathscr{C}_{2}$ of $C$ in $2_{h}^{M}$ such that if $\{X, Y\} \subset \mathscr{U}_{2}, X \subset Y$, and $Y$ is a bounded surface, then each pair of points in $\mathrm{Bd} Y$ joined by a $7 \delta_{1}$-arc in $M \backslash X$ can be joined by a $\delta_{2}$-arc in $\mathrm{Bd} Y$.

\section{Clearly}

(4) there is a neighborhood $\mathscr{C}_{3}$ of $C$ in $2_{h}^{M}$ and a $\delta_{3}>0$ such that if $X \in \mathscr{C}_{3}$ then $s\left(X, \delta_{3}, \delta_{1}\right)$.

Let $\mathscr{Q}_{4}=\left\{X \in 2_{h}^{M} \mid \rho_{h}(X, C)<(1 / 2) \delta_{3}\right\}$. By 3.3 there exist a bounded surface $N \in \bigcap_{n=1}^{4} \mathscr{U}_{n}$ and a neighborhood $\mathscr{W}_{b}$ of $C$ in $2_{h}^{M}$ such that $X \in \mathscr{C}_{5}$ implies $X \subset \operatorname{Int} N$ and $X$ is a strong deformation retract of $N$.

Let $\mathscr{W}=\bigcap_{n=1}^{5} \mathscr{W}_{n}$. Clearly $\mathscr{C}_{6}$ is a neighborhood of $C$ in $2_{n}^{n}$. Fix $X \in \mathscr{W}$. By 1.1 and 2.5 there is a bounded surface $B \in \mathscr{U}$ such that $X \subset \operatorname{Int} B$ and there is a strong deformation retraction $g: B \times I \rightarrow B$ of $B$ onto $X$ such that $g_{t}$ is an $\varepsilon / 2$-map for all $t \in I$. Thus it suffices to show the existence of a strong deformation retraction $H: N \times I \rightarrow N$ of $N$ onto $B$ such that $H_{t}$ is an $\varepsilon / 2$-map for all $t \in I$.

By choice of $\mathscr{C}_{4}$ we have $\rho_{h}(N, B)<\delta_{3}$. It follows from (4) and our choice of $\mathscr{U}_{5}$ that for all $x \in \mathrm{Bd} N$ there is a $\delta_{1}$-arc in $N$ from $x$ to some $y \in \operatorname{Bd} B$. By 1.5, each component $P$ of $\overline{N \backslash B}$ is an annulus. Let $\operatorname{Bd} P=S \cup S^{\prime}$, where $S$ and $S^{\prime}$ are boundary curves of $N$ and $B$ respectively. It follows from 1.4 that $B$ separates distinct boundary curves of $N$ in $N$. Thus

(5) for all $x \in S$, there is a $\delta_{1}$-arc $\beta$ from $x$ to some $y \in S^{\prime}$, and we may assume $\beta \backslash\{x, y\} \subset \operatorname{Int} P$.

Suppose diam $S<\delta$. By (1) and 1.6, $S$ bounds a disk of diameter less that $\delta / 2$ in $N$. Since $N$ is connected, the disk must be $N$ itself. In this case it is clear that we have a strong deformation $H: N \times$ $I \rightarrow N$ of $N$ onto $B$ such that $H_{t}$ is an $\varepsilon / 2$-map for all $t \in I$. Thus we assume

(6) $\operatorname{diam} S \geqq \delta$.

There is a set $G=\left\{x_{1}, \cdots, x_{k}\right\} \subset S$ of $k$ distinct points numbered according to an orientation of $S$ (let $x_{0}=x_{k}$ ) such that if $\alpha_{p}$ is the arc of $S$ from $x_{p-1}$ to $x_{p}$ containing no other member of $G$, then

(7) $2 \delta_{1}<\rho\left(x_{p-1}, x_{p}\right)$ and $\operatorname{diam} \alpha_{p}<5 \delta_{1}$.

By (2) and (6), $k>1$.

By (5), for each $p$ there exists $y_{p} \in S^{\prime}\left(y_{0}=y_{k}\right)$ and a $\delta_{1}-\operatorname{arc} \beta_{p}\left(\beta_{0}=\beta_{k}\right)$ in $P$ from $x_{p}$ to $y_{p}$ such that $\beta_{p} \mid\left\{x_{p}, y_{p}\right\} \subset \operatorname{Int} P$. By (7), $\beta_{p-1} \cap \beta_{p}=\phi$. 
Since $P$ is an annulus, it follows that the $\beta_{p}$ are pairwise disjoint. By choice of $B, \beta_{p-1} \cup \alpha_{p} \cup \beta_{p}$ is an arc in $M \backslash X$ from $y_{p-1} \in S^{\prime}$ to $y_{p} \in S^{\prime}$, and (7) implies

(8) $\operatorname{diam}\left(\beta_{p-1} \cup \alpha_{p} \cup \beta_{p}\right)<\delta_{1}+5 \delta_{1}+\delta_{1}=7 \delta_{1}$.

By (3), there is a $\delta_{2}$-arc $\gamma_{p}$ of $S^{\prime}$ from $y_{p-1}$ to $y_{p}$.

We claim $\gamma_{p}$ does not contain $y_{q}$ if $y_{q} \notin\left\{y_{p-1}, y_{p}\right\}$. For it follows from the disjointness of the $\beta_{p}$ that the points $y_{1}, \cdots, y_{k}$ are numbered according to an orientation of $S^{\prime}$. If some $\gamma_{p}$ contains $y_{q}$ for $y_{q} \notin$ $\left\{y_{p-1}, y_{p}\right\}$, then $\left\{y_{1}, \cdots, y_{k}\right\} \subset \gamma_{p}$. Let $x \in \alpha_{n} \neq \alpha_{p}$. Then $\rho\left(x, \gamma_{p}\right) \leqq$ $\rho\left(x, y_{n}\right) \leqq \rho\left(x, x_{n}\right)+\rho\left(x_{n}, y_{n}\right) \leqq \operatorname{diam} \alpha_{n}+\operatorname{diam} \beta_{n}<5 \delta_{1}+\delta_{1}=6 \delta_{1}$. It follows that $\operatorname{diam} S \leqq \operatorname{diam} \alpha_{p}+\operatorname{diam}\left(S \backslash \alpha_{p}\right)<5 \delta_{1}+\operatorname{diam} N_{6 \hat{o}_{1}}\left(\gamma_{p}\right) \leqq$ $5 \delta_{1}+12 \delta_{1}+\operatorname{diam} \gamma_{p}<17 \delta_{1}+\delta_{2}<\delta$ (by (3)), contrary to (6). The claim is established.

Then $L_{p}=\beta_{p-1} \cup \alpha_{p} \cup \beta_{p} \cup \gamma_{p}(p=1, \cdots, k)$ is a simple closed curve in $N$. By (8) and our choice of $\gamma_{p}$, diam $L_{p}<7 \delta_{1}+\delta_{2}$. By (1), (2), and 1.6, $L_{p}$ bounds a disk $D_{p}$ in $N$ with $\operatorname{diam} D_{p}<\varepsilon / 2$. As in the proof of $3.2, D_{p}$ is the disk of $P$ bounded by $L_{p}$.

As in 3.2, there is a strong deformation retraction $K: P \times I \rightarrow P$ of $P$ onto $S^{\prime}$ such that $K\left(D_{p} \times I\right)=D_{p}$ for all $p$. Thus $K_{t}$ is an $\varepsilon / 2$-map for all $t \in I$. As in 3.2, $K$ can be extended to a strong deformation retraction $H: N \times I \rightarrow N$ of $N$ onto $B$ such that $H_{t}$ is an $\varepsilon / 2$-map for all $t \in I$.

THEOREM 4.3. Let $\left\{A_{n}\right\}_{n=1}^{\infty}$ and $C$ be points of $2_{h}^{M} \backslash\{M\}$. Then $A_{n} \overrightarrow{o_{h}} C$ if and only if there exists $N \in 2_{h}^{M}$ such that each component of $\stackrel{\rho_{h}}{N}$ is a bounded surface and $A_{n} \underset{D(N)}{\longrightarrow}$.

Proof. By 3.3, there is a compact 2-manifold with boundary $N \in 2_{h}^{M}$ and a neighborhood $\mathscr{C}$ of $C$ in $2_{h}^{M}$ such that if $X \in \mathscr{Q} b$ then $X \subset \operatorname{Int} N$ and $X$ is a strong deformation retract of $N$.

Suppose $A_{n} \underset{\rho_{h}}{\rightarrow} C$. Let $\varepsilon>0$. By 4.2 there is a compact 2manifold with boundary $B \in \mathscr{C}$ and a neighborhood $\mathscr{V}$ of $C$ in $2_{h}^{H}$ with $\mathscr{V} \subset \mathscr{U}$ such that if $X \in \mathscr{Y}$ then $X \subset \operatorname{Int} B$ and there is an $\varepsilon / 2$-map $r: B \rightarrow B$ that is a strong deformation retraction of $B$ onto $X$. Choose an $m$ such that $n>m$ implies $A_{n} \in \mathscr{Y}$.

Let $f: N \rightarrow N$ be a deformation retraction of $N$ onto $B$. Let $f_{n}: B \rightarrow B$ be an $\varepsilon / 2$-map that is a deformation retraction of $B$ onto $A_{n}$ for $n>m$. Let $f_{0}: B \rightarrow B$ be an $\varepsilon / 2$-map that is a deformation retraction of $B$ onto $C$. Define $r_{n}: N \rightarrow N$ for $n=0, n>m$ by $r_{n}(x)=$ $f_{n}(f(x))$. For all $x \in N$ and $n>m, \rho\left(r_{n}(x), r_{0}(x)\right)<\varepsilon$. Hence $A_{n} \underset{D(N)}{\longrightarrow} C$.

Conversely, suppose $A_{n} \underset{D(N)}{\longrightarrow} C$. There exist deformation retractions $r_{n}: N \rightarrow N$ of $N$ onto $A_{n^{\prime}}, r_{0}: N \rightarrow N$ of $N$ onto $C$ such that $r_{n} \rightarrow r_{0}$ uniformly on $N$. 
If $x \in C, \rho\left(x, r_{n}(x)\right) \rightarrow \rho\left(x, r_{0}(x)\right)=0$. Hence $\rho\left(x, A_{n}\right) \rightarrow 0$.

If $x_{n} \in A_{n}, \rho\left(x_{n}, r_{0}\left(x_{n}\right)\right)=\rho\left(r_{n}\left(x_{n}\right), r_{0}\left(x_{n}\right)\right) \rightarrow 0$. Hence $\rho\left(x_{n}, C\right) \rightarrow 0$. We conclude $A_{n} \underset{\rho_{s}}{\rightarrow} C$.

Let $\varepsilon>0$. Let $\delta>0$ be such that if $\{x, y\} \subset N$ and $\rho(x, y)<\delta$ then $\rho\left(r_{0}(x), r_{0}(y)\right)<\varepsilon / 6$. Let $\delta^{\prime}>0$ be such that $s\left(N, \delta^{\prime}, \delta\right)$. Let $m>0$ be such that $n>m$ implies that for all $x \in N, \rho\left(r_{n}(x), r_{0}(x)\right)<$ $\varepsilon / 6$.

If $\{x, y\} \subset N, \rho(x, y)<\delta$, and $n>m$, then $\rho\left(r_{n}(x), r_{n}(y)\right) \leqq \rho\left(r_{n}(x)\right.$, $\left.r_{0}(x)\right)+\rho\left(r_{0}(x), r_{0}(y)\right)+\rho\left(r_{0}(y), r_{n}(y)\right)<\varepsilon / 6+\varepsilon / 6+\varepsilon / 6=\varepsilon / 2$.

Let $K \subset A_{n} \subset N$, diam $K<\delta^{\prime}$. There is a contraction $h: K \times I \rightarrow N$ of $K$ to a point such that $\operatorname{diam} h(K \times I)<\delta$. Therefore, for $n>m$, $r_{n} \circ h: K \times I \rightarrow N$ is a contraction of $K$ to a point such that $r_{n} \circ h(K \times I) \subset A_{n}$ and $\operatorname{diam}\left(r_{n} \circ h(K \times I)\right)<\varepsilon / 2+\varepsilon / 2=\varepsilon$. Hence $s\left(A_{n}, \delta^{\prime}, \varepsilon\right)$ for $n>m$, so $A_{n} \underset{o_{h}}{\longrightarrow} C$.

\section{THEOREM 4.4. $2_{n}^{M}$ is an ANR ( $\left./ \mathscr{C}\right)$.}

Proof. If $N$ and $\mathscr{Z}$ are as above, the previous theorem implies the inclusion of the set $\mathscr{C}$ into $D(N)$ is an open embedding. Since $D(N)$ is an ANR ( $\mathscr{C})([11], 5.5$, p. 389), it follows ([9], 3.1, p. 391) that $\mathscr{U}$ is an $\operatorname{ANR}(\mathscr{C})$. Since $M$ is an isolated point of $2_{h}^{M}$ (because

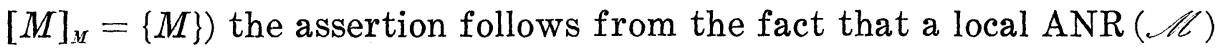
is an $\operatorname{ANR}(\mathscr{C l})$ ([9], 3.3, p. 392).

TheOREM 4.5. Let $A R_{h}^{I I}=\left\{X \in 2_{h}^{I I} \mid X\right.$ is an AR $\}$. Then $A R_{h}^{I I}$ is a component of $2_{h}^{M}$.

Proof. Since $A R_{h}^{M}$ is the set of all members of $2_{h}^{M}$ with the homotopy type of a point, $A R_{h}^{M}$ is open and closed in $2_{h}^{M}$, and thus is a union of components of $2_{h}^{M}$. We must show $A R_{h}^{M}$ is connected.

Let $C_{n} \in A R_{h}^{M}(n=0,1)$. By 3.2 there is an arc in $A R_{h}^{M}$ from $C_{n}$ to $N_{n}$, where $N_{n}$ is a disk. Let $p_{n} \in N$ and let $h^{n}: N_{n} \times I \rightarrow N_{n}$ be a pseudoisotopy of $N_{n}$ onto $p_{n}$. Then (using 1.3) $\left\{h^{n}\left(N_{n} \times\{t\}\right) \mid t \in I\right\}$ contains an arc in $A R_{h}^{M}$ from $N_{n}$ to $\left\{p_{n}\right\}$. Let $h: I \rightarrow M$ be a map such that $h(0)=p_{0}$ and $h(1)=p_{1}$. By 1.3, $\{\{h(t)\} \mid t \in I\}$ contains an arc in $A R_{h}^{M}$ from $\left\{p_{0}\right\}$ to $\left\{p_{1}\right\}$. Thus there is an arc in $A R_{h}^{M}$ from $C_{0}$ to $C_{1}$.

THEOREM 4.6. $A R_{h}^{M}=L(M)$ as topological spaces.

Proof. Clearly they are equal as sets. Let $C \in A R_{h}^{M}$. As above, there is a disk $N \subset M$ such that $C \subset \operatorname{Int} N$ and $C$ is a strong deformation retract of $N$. We know $A_{n} \underset{\rho_{h}}{\longrightarrow} C$ if and only if $A_{n} \underset{D(N)}{\longrightarrow} C$. 
But $A_{n} \underset{D(N)}{\longrightarrow} C$ if and only if $A_{n} \underset{L(M)}{\longrightarrow} C([11], 5.4$, p. 388).

Clearly the map $j: M \rightarrow A R_{h}^{M}$ defined by $j(x)=\{x\}$ is an embedding. We have the following:

COROLLARY 4.7. $j(M)$ is a deformation retract of $A R_{h}^{M}$. Thus $A R_{h}^{M}$ has the same homotopy type as $M$.

Proof. This follows from Theorem 4.6 and ([11], 5.5, p. 389).

\section{REFERENCES}

1. B. J. Ball and Jo Ford, Spaces of ANR's, Fund. Math., 77 (1972), 33-49.

2. - Spaces of ANR's. II, Fund. Math., 78 (1973), 209-216.

3. K. Borsuk, On some metrizations of the hyperspace of compact sets, Fund. Math., 41 (1954), 168-202.

4. - Theory of Retracts, Polish Scientific Publishers, Warsaw, 1967.

5. L. Boxer, The Space of Absolute Neighborhood Retracts of a Closed Surface, Ph. D. Thesis, University of Illinois, 1976.

6. M. M. Cohen, A Course in Simple-Homotopy Theory, Springer-Verlag, New York, 1973.

7. J. Dugundji, Topology, Allyn and Bacon, Boston, 1966.

8. D. B. A. Epstein, Curves on 2-manifolds and isotopies, Acta Math., 115 (1966), 83107.

9. O. Hanner, Some theorems on absolute neighborhood retracts, Arkiv for Matematik, 1 (1951), 389-408.

10. A. G. Kurosh, The Theory of Groups, vol. II, Chelsea, New York, 1956.

11. N. R. Wagner, A continuity property with applications to the topology of 2manifolds, Trans. Amer. Math. Soc., 200 (1974), 369-393.

12. J. H. C. Whitehead, On the homotopy type of ANR's, Bull., Amer. Math. Soc., 54 (1948), 1113-1145,

13. G. T. Whyburn, Analytic Topology, Amer. Math. Soc., Providence, 1942.

Received April 26, 1977 and in revised form January 5, 1978.

University of Georgia

Current address: Salem College

Winston-Salem, NC 27108 


\section{PACIFIC JOURNAL OF MATHEMATICS}

EDITORS

RICHARD ARENS (Managing Editor)

University of California

Los Angeles, California 90024

C. W. Curtis

University of Oregon

Eugene, OR 97403

C. C. MOORE

University of California

Berkeley, CA 94720
J. DUGUNDJI

Department of Mathematics University of Southern Californı Los Angeles, California 90007

R. Finn and J. Milgram Stanford University Stanford, California 94305

\section{ASSOCIATE EDITORS}

E. F. BECKENBACH

B. H. NEUMANN

F. WOLF

K. YoSHIDA

\section{SUPPORTING INSTITUTIONS}

UNIVERSITY OF BRITISH COLUMBIA
CALIFORNIA INSTITUTE OF TECHNOLOGY
UNIVERSITY OF CALIFORNIA
MONTANA STATE UNIVERSITY
UNIVERSITY OF NEVADA, RENO
NEW MEXICO STATE UNIVERSITY
OREGON STATE UNIVERSITY
UNIVERSITY OF OREGON

UNIVERSITY OF SOUTHERNEALIFORNIA STANFORD UNIVERSITY UNIVERSITY OF HAWAII UNIVERSITY OF TOKYO UNIVERSITY OF UTAH WASHINGTON STATE UNIVERSITY UNIVERSITY OF WASHINGTON 


\section{Pacific Journal of Mathematics \\ Vol. 79 , No. 1 \\ May, 1978}

Teófilo Abuabara, A remark on infinitely nuclearly differentiable

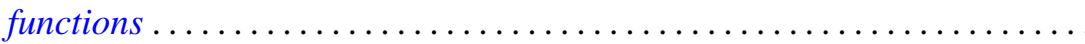

David Fenimore Anderson, Projective modules over subrings of $k[X, Y]$

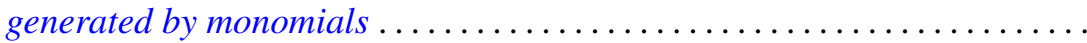

Joseph Barback and Thomas Graham McLaughlin, On the intersection of

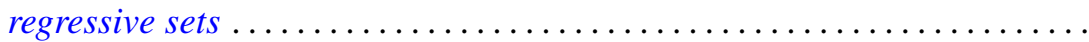

Murray Bell, John Norman Ginsburg and R. Grant Woods, Cardinal inequalities for topological spaces involving the weak Lindelof

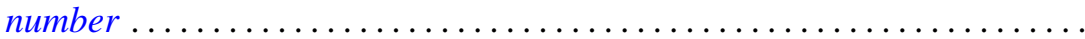

Laurence Richard Boxer, The space of ANRs of a closed surface ............

Zvonko Cerin, Homotopy properties of locally compact spaces at

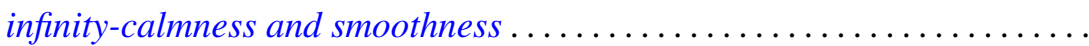

Isidor Fleischer and Ivo G. Rosenberg, The Galois connection between partial functions and relations..................................

John R. Giles, David Allan Gregory and Brailey Sims, Geometrical implications of upper semi-continuity of the duality mapping on a Banach

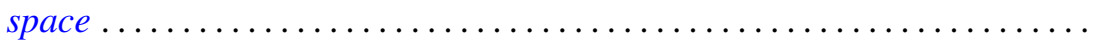

Troy Lee Hicks, Fixed-point theorems in locally convex spaces ............ Hugo Junghenn, Almost periodic functions on semidirect products of transformation semigroups ........................

Victor Kaftal, On the theory of compact operators in von Neumann algebras. II . . . .

Haynes Miller, A spectral sequence for the homology of an infinite

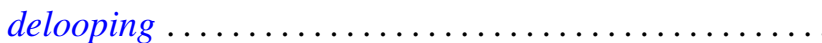

Sanford S. Miller, Petru T. Mocanu and Maxwell O. Reade, Starlike integral operators...

Stanley Stephen Page, Regular FPF rings ...............

Ghan Shyam Pandey, Multipliers for C, 1 summability of Fourier series ...

Shigeo Segawa, Bounded analytic functions on unbounded covering surfaces...

Steven Eugene Shreve, Probability measures and the C-sets of

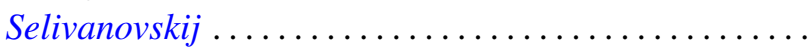

Tor Skjelbred, Combinatorial geometry and actions of compact Lie groups....

Alan Sloan, A note on exponentials of distributions.

Colin Eric Sutherland, Type analysis of the regular representation of a nonunimodular group.

Mark Phillip Thomas, Algebra homomorphisms and the functional

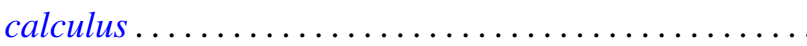

Sergio Eduardo Zarantonello, A representation of $H^{p}$-functions with

$0<p<\infty$. 